\author{
UNIVERSIDADE DE SÃO PAULO \\ ESCOLA DE ENFERMAGEM DE RIBEIRÃO PRETO
}

CRISTIANE THOMAZ DE AQUINO EXEL ANDRADE

\title{
Pacientes com metástases vertebrais submetidos à cirurgia - avaliação da Qualidade de Vida Relacionada à Saúde
}

Ribeirão Preto 2015 


\section{Pacientes com metástases vertebrais submetidos à cirurgia- avaliação da Qualidade de Vida Relacionada à Saúde}

Dissertação apresentada à Escola de Enfermagem de Ribeirão Preto da Universidade de São Paulo, para obtenção do título de Mestre em Ciências, Programa Enfermagem Fundamental.

Linha de Pesquisa: Processo de cuidar do adulto com doenças agudas e crônico-degenerativas.

Orientadora: Profa. Dra.Namie Okino Sawada.

Ribeirão Preto

2015 
Autorizo a reprodução e divulgação total ou parcial deste trabalho, por qualquer meio convencional ou eletrônico, para fins de estudo e pesquisa, desde que citada a fonte.

FICHA CATALOGRÁFICA

Andrade, Cristiane Thomaz de Aquino Exel

Pacientes com metástases vertebrais submetidos à cirurgia - avaliação da Qualidade de Vida Relacionada à Saúde. Ribeirão Preto, 2015.

80 p. : il. ; $30 \mathrm{~cm}$

Dissertação de Mestrado, apresentada à Escola de Enfermagem de Ribeirão Preto/USP. Área de concentração: Enfermagem Fundamental.

Orientadora: Sawada, Namie Okino.

1. Metástase vertebral. 2. Qualidade de Vida. 3. Neoplasias.

4. Cirurgia Paliativa. 5. Capacidade funcional. 


\section{FOLHA DE APROVAÇÃO}

Nome: ANDRADE, Cristiane Thomaz de Aquino Exel

Título: Pacientes com metástases vertebrais submetidos à cirurgia - avaliação da Qualidade de Vida Relacionada à Saúde.

Dissertação apresentada à Escola de Enfermagem de Ribeirão Preto da Universidade de São Paulo, para obtenção do título de Mestre em Ciências, Programa Enfermagem Fundamental.

Aprovado em: / 2015

BANCA EXAMINADORA

Prof. Dr.(a)

Instituição: Assinatura:

Prof. Dr.(a)

Instituição: Assinatura:

Prof. Dr.(a) Instituição: Assinatura: 
"A mente que se abre para uma nova ideia jamais voltará ao seu tamanho original.” Albert Einstein 


\section{AGRADECIMENTOS}

“Cada pessoa que passa em nossa vida, passa sozinha, é porque cada pessoa é única e nenhuma substitui a outra! Cada pessoa que passa em nossa vida passa sozinha e não nos deixa só porque deixa um pouco de si e leva um pouquinho de nós. Essa é a mais bela responsabilidade da vida e a prova de que as pessoas não se encontram por acaso." 
Primeiramente, agradeço a Deus por me amparar nos momentos difíceis, me dar força interior para superar as dificuldades, mostrar o caminho nas horas incertas e me suprir em todas as minhas necessidades.

À minha Família, a qual amo muito, pelo carinho, paciência e incentivo.

Ao meu companheiro de todas as horas, Diocésio, o meu porto seguro, exemplo de ser humano e profissional, pela sua compreensão, respeito, tolerância, por presentear-me com a família que sempre sonhei e por todas as atitudes que o faz merecedor do meu amor...Te amo!!!

À Letícia, minha filha tão amada, obrigada por me ensinar todos os dias como ser mais paciente e me permitir desfrutar desse amor tão puro e verdadeiro...Te amo!!! 


\section{AGRADECIMENTO ESPECIAL}

À minha orientadora, Namie Okino Sawada, por acreditar que eu era capaz e pela orientação. Mesmo sem me conhecer direito, você abriu as portas, como uma mãe abre os braços para receber um filho. Só tenho a agradecer aos seus ensinamentos (pessoais e acadêmicos), orientações, palavras de incentivo, puxões de orelha, paciência e dedicação....Muito obrigada!

À minha companheira de ambulatório, Lílian Maria Pacola, muito obrigada pelos ensinamentos, paciência e disponibilidade.

Ao Prof. Dr. Helton Luiz Aparecido Defino, por ter aberto as portas do ambulatório de coluna para a realização desta pesquisa.

E a todos os Participantes, que mesmo atravessando um momento delicado da vida, me acolheram com um sorriso. 


\section{RESUMO}

ANDRADE, C.T.A.E. Pacientes com metástases vertebrais submetidos à cirurgia avaliação da Qualidade de Vida Relacionada à Saúde. 2015. 80 f. Dissertação (Mestrado) - Escola de Enfermagem de Ribeirão Preto, Universidade de São Paulo, Ribeirão Preto, 2015.

A dor e a incapacidade funcional decorrente do comprometimento da coluna vertebral por metástases impactam de forma significativa na qualidade de vida de qualquer paciente. Como forma de amenizar os sintomas, dois grandes grupos de tratamento são possíveis na atualidade: cirurgia e/ou radioterapia. O objetivo principal deste estudo foi avaliar o impacto da cirurgia de descompressão medular na Qualidade de Vida Relacionada à Saúde de pacientes acometidos por metástase em algum nível da coluna vertebral. Trata-se de um estudo quantitativo, descritivo, correlacional e de corte longitudinal do tipo prospectivo que englobará quatro avaliações: antes da cirurgia (T0), dois (T1), quatro (T2) e seis (T3) meses após o procedimento cirúrgico. As entrevistas individuais foram realizadas pela pesquisadora no serviço de internação no momento anterior à cirurgia e no ambulatório nos três momentos subsequentes acima descritos. Os instrumentos utilizados para coleta de dados foram: questionário estruturado para caracterização sociodemográfica e clínica da amostra; Escala de Dor de Faces Revisada (FPS-R); questionário de avalição da Qualidade de Vida Relacionada à Saúde (EORTC QLQ-C30). Realizou-se comparação dos resultados do pré-operatório (T0) com os momentos T1, T2 e T3. A amostra foi constituída por 22 pacientes, com idade média de 56 anos, sem diferenças quanto à prevalência relacionada ao sexo, todos com baixa escolaridade e com renda mensal média de aproximadamente um salário mínimo. Os tumores primários mais prevalentes por ordem de frequência foram: mama, próstata, pulmão e colorretal. A abordagem cirúrgica mais utilizada foi a vertebral posterior (63,6\%), sendo o segmento lombar $(36,4 \%)$ o mais acometido. No período pré-operatório (T0), os participantes apresentaram baixo escore para Estado de Saúde Global/Qualidade de Vida e para quase todos os domínios da Escala Funcional e Escala de Sintomas. Nos três momentos distintos (T1, T2 e T3) comparados em relação ao pré-operatório (T0), houve melhora estatisticamente significativa no desempenho de papel, na presença de dor, insônia e constipação intestinal dos pacientes. Não houve diferença significativa nas atividades de vida diária em nenhum dos três períodos analisados após a cirurgia, sempre levando-se em consideração a fase pré-operatória (T0) como padrão.

Palavras-chave: metástase vertebral, Qualidade de Vida, neoplasias, cirurgia paliativa, capacidade funcional. 


\begin{abstract}
ANDRADE, C.T.A.E. Patients with vertebral metastasis underwent surgery- evaluation of Health-Related Quality of Life. 2015. 80 f. Master's Thesis - University of São Paulo School of Nursing, Ribeirão Preto, 2015.

Pain and functional disability resulting from the impairment of spinal metastasis impact significantly on Quality of Life of any patient. To reduce the symptoms, two groups of treatment are possible nowadays: surgery and/or radiotherapy. The aim of this study was to evaluate the impact of spinal decompressive surgery on Health-Related Quality of Life (HRQoL) of patients affected by metastasis at some level of the spine. It is a prospective, quantitative, descriptive correlational and longitudinal study that comprises four ratings: before surgery (T0), two (T1), four (T2) and six (T3) months after surgical procedure. The researcher held individual interviews at the hospital service prior to surgery and at the ambulatory within three moments described above. The following data collection instruments were used: structured questionnaire for sociodemographic and clinical sample characteristics; Faces Pain Scale Revised (FPS-R); questionnaire of Health-Related Quality of Life (EORTC QLQ-C30). It was performed comparison the preoperative (T0) results with times T1, T2 and T3. The sample consisted of 22 patients with a mean age of 56 years without differences in prevalence related to sex, all with low education and with average monthly income of approximately one basic wage. The most prevalent primary tumors were in order of frequency: breast, prostate, lung and colorectal. The most used surgical approach was the posterior vertebral $(63,6 \%)$ in which the lumbar segment $(36,4 \%)$ was the most affected.

In the preoperative period (T0), participants presented low score for State of Global Health/Quality of Life and for most areas of Functional Scale and Symptoms Scale. In three different times (T1, T2 and T3) compared regarding the preoperative period (T0), there was a statistically significant improvement in the performance status, presence of pain, insomnia and constipation of patients. There was no significant difference in daily activities in any of the three periods analyzed after surgery, always taking into account the preoperative phase (T0) as standard.
\end{abstract}

Key words: vertebral metastasis, Quality of Life, neoplasias, palliative surgery, functional capacity. 


\section{RESUMEN}

ANDRADE, C.T.A.E. Pacientes com metástasis vertebral sometidos a cirurgía evaluación de la Calidad de Vida Relacionada a la Salud. 2015. 80 f. Disertación (Maestría) - Escuela de Enfermería de Ribeirão Preto, Universidad de São Paulo, Ribeirão Preto, 2015.

El dolor y la discapacidad funcional resultante de la acometimiento de la columna vertebral por metástasis impactan significativamente la calidad de vida de cualquier paciente. Con el fin de reducir los síntomas, hay dos grandes grupos posibles de tratamiento hoy en día: la cirugía y / o radioterapia. El objetivo de este estudio ha sido evaluar el impacto de la cirugía de descompresión espinal en la Calidad de Vida Relacionada con la Salud de los pacientes afectados por metástasis en algún nivel de la columna vertebral. Se trata de un estudio prospectivo, cuantitativo, descriptivo, correlacional y longitudinal que comprende cuatro clasificaciones distintas: antes de la cirugía (T0), dos (T1), cuatro (T2) y seis meses (T3) luego de la cirugía. Las entrevistas individuales se llevaron a cabo por el investigador en el servicio hospitalario antes de la cirugía y en la clínica en los tres momentos descritos anteriormente. Los instrumentos utilizados para la recolección de datos fueron: cuestionario estructurado para la caracterización sociodemográfica y clínica de la muestra; Escala de Dolor Facial Revisada (FPS-R); encuesta de calidad de vida relacionada con la salud (EORTC QLQC30). Se ha realizado la comparación de los resultados preoperatorios (T0) con los tiempos T1, T2 y T3. La muestra consistió en 22 pacientes con una edad media de 56 años, sin diferencias en la prevalencia relacionada al sexo, todas con bajo nivel de educación y con un ingreso mensual promedio de aproximadamente un salario mínimo. Los tumores primarios más frecuentes fueron, en orden de frecuencia: de mama, próstata, pulmón y colorrectal. El abordaje quirúrgico más utilizado fue la vertebral posterior (63,6\%), y el segmento lumbar (36,4\%) el más afectado. En el preoperatorio (T0), los participantes tuvieron puntuación baja de Estado de Salud Global / Calidad de Vida y en casi todas las áreas de la Escala Funcional y de la Escala de Síntomas. En los tres momentos en comparación (T1, T2 y T3) en relación con el período preoperatorio (T0), hubo una mejoría, desde el punto de vista estadístico, significativa en el rendimiento de rol, en la presencia de dolor, insomnio y estreñimiento de los pacientes. No hubo diferencias significativas en las actividades diarias en cualquiera de los tres períodos analizados después de la cirugía, siempre teniendo en cuenta la fase de preoperatoria (T0) como patrón.

Palabras-clave: metástasis vertebral, Calidad de Vida, neoplasías, cirugía paliativa, capacidad funcional. 


\section{LISTA DE QUADROS}

Quadro 1 - Escores do EORTC QLQ-C30 Versão 3.0 ......................................................... 36 


\section{LISTA DE ILUSTRAÇÕES}

Figura 1 - Escala de Likert de quatro pontos - EORTC QLQ-C30 ..................................... 36

Figura 2 - Escala de Likert de sete pontos - EORTC QLQ-C30 ......................................... 37 


\section{LISTA DE TABELAS}

Tabela 1 - Frequência e porcentagem das características sociodemográficas dos pacientes ( $\mathrm{n}=22)$ com metástase vertebral do Hospital das Clínicas da Faculdade de Medicina de Ribeirão Preto - USP, 2013-2015.

Tabela 2 - Frequência e porcentagem das características clínicas dos pacientes $(n=22)$ com metástase vertebral do Hospital das Clínicas da Faculdade de Medicina de Ribeirão Preto - USP, 2013-2015.

Tabela 3 - Média e desvio-padrão dos escores das escalas do instrumento EORTC QLQC30 apresentados pelos pacientes $(n=22)$ com metástase vertebral, do Hospital das Clínicas da Faculdade de Medicina de Ribeirão Preto-USP, nos tempos avaliados (T0, T1, T2 e T3), 2013-2015.

Tabela 4 - Média e desvio-padrão da diferença entre os pares de avaliação do momento T0 e o T1, valor p do Teste $t$ de Student pareado dos escores do instrumento EORTC QLQ-C30 apresentados pelos pacientes $(n=17)$ com metástase vertebral, do Hospital das Clínicas da Faculdade de Medicina de Ribeirão Preto-USP, 2013-2015.

Tabela 5 - Média e desvio-padrão da diferença entre os pares de avaliação do momento T0 e o T2, valor p do Teste $t$ de Student pareado dos escores do instrumento EORTC QLQ-C30 apresentados pelos pacientes $(n=14)$ com metástase vertebral, do Hospital das Clínicas da Faculdade de Medicina de Ribeirão Preto-USP, 2013-2015.

Tabela 6 - Média e desvio-padrão da diferença entre os pares de avaliação do momento T0 e o T3, valor p do Teste $t$ de Student pareado dos escores do instrumento EORTC QLQ-C30 apresentados pelos pacientes $(\mathrm{n}=12)$ com metástase vertebral, do Hospital das Clínicas da Faculdade de Medicina de Ribeirão Preto-USP, 2013-2015.

Tabela 7 - Frequência e porcentagem da Escala de Dor de Faces Revisada (FPS-R) dos pacientes $(\mathrm{n}=22)$ com metástase vertebral, do Hospital das Clínicas da Faculdade de Medicina de Ribeirão Preto-USP, 2013-2015.

Tabela 8 - Análise descritiva da variável dor do instrumento EORTC QLQ-C30, da Escala de Dor de Faces Revisada (FPS-R) e do Índice de Barthel dos pacientes $(n=22)$ com metástase vertebral, do Hospital das Clínicas da Faculdade de Medicina de Ribeirão Preto-USP, 2013-2015.

Tabela 9 - Valor de p do Teste t de Student pareado dos escores da dor avaliada por duas escalas (EORTC QLQ-C30 e FPS-R) e da capacidade funcional (Índice de Barthel) comparando o (T0) com os três tempos subsequentes T1 $(n=17), \mathbf{T} 2(n=14)$ e T3 $(n=12)$ dos pacientes com metástase vertebral, do Hospital das Clínicas da Faculdade de Medicina de Ribeirão Preto-USP, 2013-2015. 


\section{LISTA DE SIGLAS}

AVA

AVDs

AVP

CEP

CNS

DP

ECOG Performance Status

EORTC QLQ-C30

ESG/QV

FACT-G

FPS-R

HC-FMRP

IARC

INCA

OMS

QV

QVRS

SF-36

SPSS

SUS

TCLE

T0

$\mathrm{T} 1$

$\mathrm{T} 2$

T3

USP

WCPT
Abordagem Vertebral Anterior

Atividades de Vida Diária

Abordagem Vertebral Posterior

Comitê de Ética em Pesquisa

Conselho Nacional de Saúde

Desvio-padrão

Eastern Cooperative Oncology Group

European Organization for Research and Treatment of Cancer Quality of Life Questionnaire "core" 30 item

Estado de Saúde Global e Qualidade de Vida

Functional Assessment of Cancer Therapy-General

Escala de Dor de Faces Revisada

Hospital das Clínicas da Faculdade de Medicina de Ribeirão Preto

International Agency for Research on Cancer

Instituto Nacional do Câncer

Organização Mundial da Saúde

Qualidade de Vida

Qualidade de Vida Relacionada à Saúde

Short-Form Health Survey

Statistical Package for the Social Sciences

Sistema Único de Saúde

Termo de Consentimento Livre e Esclarecido

Pré-operatório

Dois meses após a cirurgia

Quatro meses após a cirurgia

Seis meses após a cirurgia

Universidade de São Paulo

World Confederation for PhysicalTherapy 


\section{SUMÁRIO}

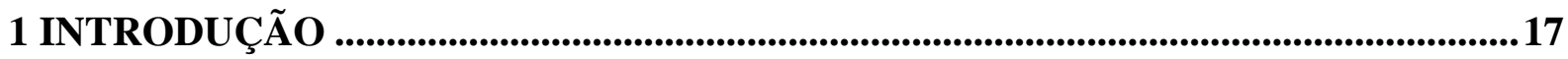

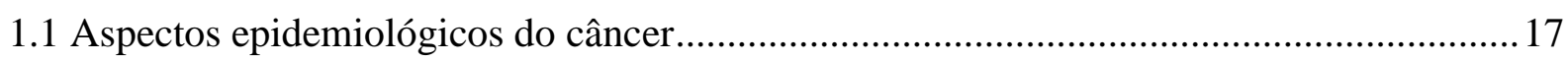

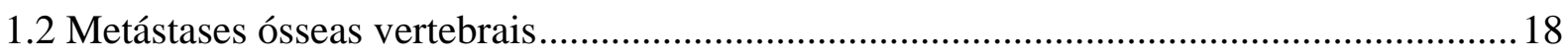

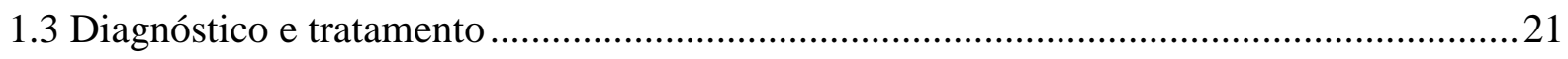

1.4 Qualidade de vida relacionada à saúde (QVRS) e o papel da fisioterapia .........................24

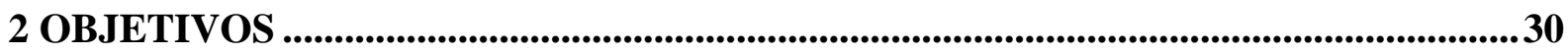

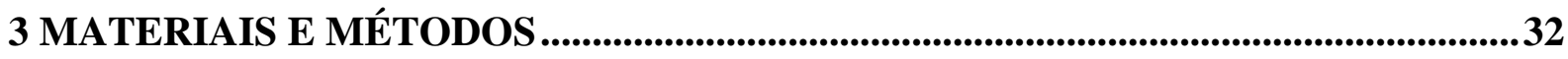

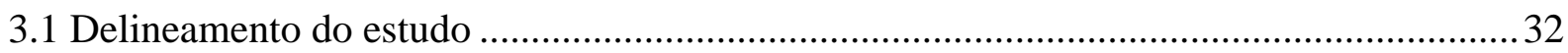

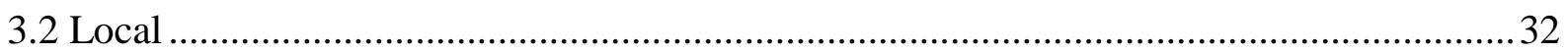

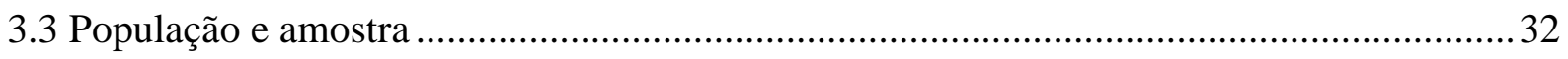

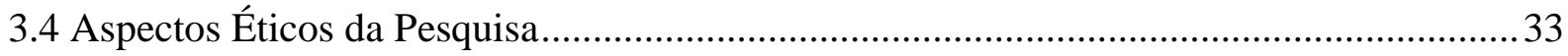

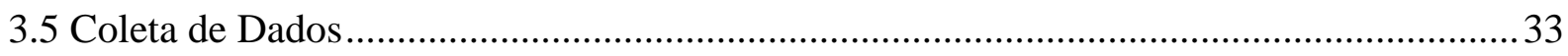

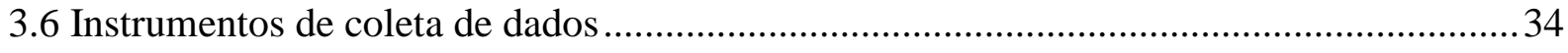

3.6.1 Instrumento para caracterização sociodemográfica e clínica ..........................................34

3.6.2 Escala de Dor de Faces Revisada (FPS-R) .................................................................. 35

3.6.3 Instrumento de avaliação da qualidade de vida relacionada à saúde - EORTC QLQ-

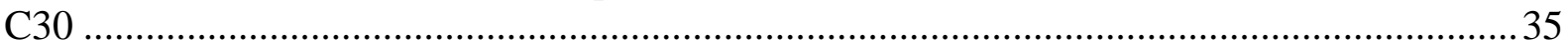

3.6.4 Instrumento para avaliar a capacidade funcional - Índice de Barthel .............................37

4 PROCESSAMENTO E ANÁLISE DOS DADOS ..........................................................39

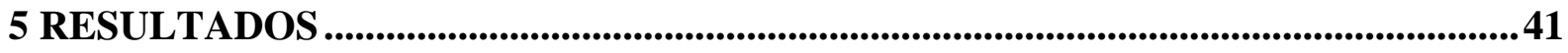

5.1 Caracterização sociodemográfica dos pacientes com metástase vertebral .........................41

5.2 Caracterização clínica dos pacientes com metástase vertebral..........................................43

5.3 Avaliação da Qualidade de Vida Relacionada à Saúde dos pacientes com metástase vertebral (dimensões do EORTC QLQ-C30) ...................................................................... 44

6 DISCUSSÃO

7 CONCLUSÃO

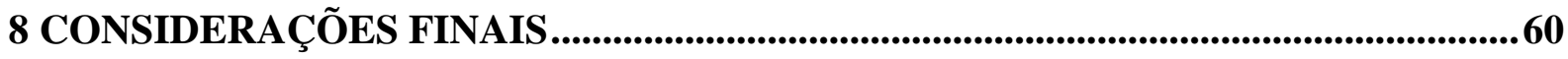

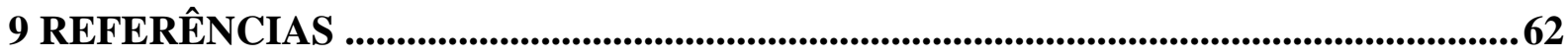

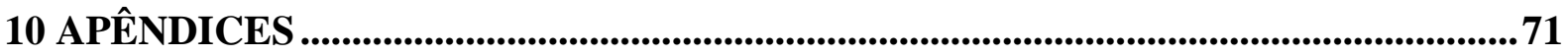

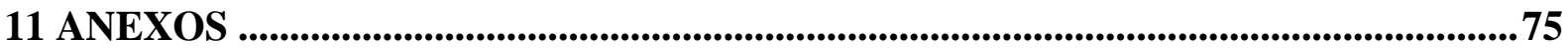


INTRODUÇÃO 


\section{INTRODUÇÃO}

\subsection{Aspectos epidemiológicos do câncer}

O câncer tem sido reconhecido pela sociedade como uma epidemia que aflige a humanidade, sendo esclarecido cientificamente como um dos grandes desafios da medicina. Com uma história de divergências em relação ao entendimento da doença e seu combate, mesmo diante de inovações tecnológicas terapêuticas, até o momento não se alcançou a cura para todas as formas da doença. Isso a torna um grave e importante problema de saúde pública mundial (TEIXEIRA; FONSECA, 2007).

De acordo com estimativas mundiais do projeto Globocan 2012, da Agência Internacional para Pesquisa em Câncer (Iarc, InternationalAgency for Research on Cancer) e da Organização Mundial da Saúde (OMS), houve 14,1 milhões de casos novos e um total de 8,2 milhões de mortes por câncer, em todo o mundo, em 2012 (IARC, 2014). Historicamente as maiores taxas de incidência de câncer eram encontradas em países desenvolvidos. Em 2010 esta tendência mostrou a sua primeira inversão, pois dos dez milhões de casos novos diagnosticados naquele ano, cinco milhões e meio foram em países em desenvolvimento (WHO, 2011).

É estimado pela organização mundial de saúde que no ano 2030 o câncer alcance aproximadamente 27 milhões de casos novos, 17 milhões de óbitos e 75 milhões de pessoas com diagnóstico de qualquer tipo de câncer, com maior impacto em países de baixa e média renda. Nos países desenvolvidos os tumores mais frequentes na população masculina são próstata, pulmão e cólon/reto e na população feminina os de mama, cólon/reto e pulmão. Já nos países em desenvolvimento há uma mudança brusca na incidência, sendo que nos homens os tumores mais prevalentes são pulmão, próstata e estômago e nas mulheres os de mama, colo do útero e pulmão (INCA, 2014).

Especificamente no Brasil, as mudanças no perfil epidemiológico da população nos últimos 50 anos contribuíram muito para o avanço do câncer como uma epidemia. As doenças infecciosas e parasitárias eram estatisticamente reconhecidas como a primeira causa de morte no país, sendo que atualmente foram ultrapassadas pelas doenças cardiovasculares e pelas doenças malignas. Além disso, com os avanços tecnológicos e científicos e o crescente desenvolvimento industrial e urbano houve um aumento importante na expectativa de vida da 
população contribuindo indiretamente para o aumento na incidência das neoplasias (RODRIGUES, 2010).

Também denominado de neoplasia ou tumor maligno, o câncer representa um amplo grupo de doenças que pode afetar qualquer área corporal, sem causa exata definida, manifestada por uma sequência de eventos. A carcinogênese, etapa de formação de um tumor, ocorre lentamente e envolve um longo processo de alterações nos mecanismos de regulação da proliferação, diferenciação e apoptose celular. Este processo leva a um crescimento desordenado, descontrolado e irreversível de células anormais, podendo culminar com o aparecimento de metástases que acometem partes adjacentes do corpo ou invadem outros órgãos, sendo a principal causa de mortalidade desta doença (INCA, 2014; WHO, 2011; CANPONERO, 2008).

O número de sobreviventes de câncer aumenta, anualmente, sobretudo devido aos avanços nos tratamentos médicos disponíveis e ao constante envelhecimento da população (COLEMAN et al., 2011). Destaca-se também um aumento de indivíduos metastáticos que apresentam uma maior sobrevida global quando comparado às décadas passadas (TREANOR; DONNELLY, 2012). Contudo, o objetivo da ciência é alcançar cada vez mais estes avanços, sem deixar de proporcionar uma qualidade de vida digna aos pacientes.

\subsection{Metástases ósseas vertebrais}

A origem da palavra metástase vem do grego mestastasis e tem como significado mudança de lugar, transferência. Em 1829, Recamier descreveu o termo como sendo uma deposição à distância de um tumor primário (LAUFER; MAÇANEIRO; MIYAMOTO, 2004).

Metástase é a disseminação do câncer de seu sítio primário para alguma parte do corpo humano. Sua origem ocorre através da migração das células metastáticas na corrente sanguínea ou no sistema linfático (MACCAURO et al., 2011).

Muitas células que migram do sítio primário morrem sem causar problemas. Algumas se estabelecem em uma nova área, onde começam a crescer e formar novos tumores. Em alguns casos, os tumores metastáticos são encontrados quando o sítio primário já foi diagnosticado; já em outros, a metástase é descoberta primeiro; raramente ocorrem as 
metástases sem correlação com um câncer primário, sendo considerado um tumor de primário oculto ou desconhecido (HOSONO et al., 1995).

O tecido esquelético é o terceiro local mais frequente de lesões secundárias neoplásicas, sendo superado pelas lesões hepáticas e pulmonares (STEINMETZ; MEKHAIL; BENZEL, 2001). Os tumores primários responsáveis pelo desenvolvimento de metástases ósseas são por ordem de incidência: próstata, mama, pulmão, rim e tireoide. Devido à elevada prevalência do carcinoma de mama, próstata e pulmão, estes são responsáveis por mais de 80\% dos casos (MACCAURO et al., 2011).

A maior incidência de lesões metastáticas ósseas ocorre no esqueleto axial. A coluna vertebral é o local mais frequente, com taxas de prevalência variando entre 30 a 80\% (WEDIN; BAUER; RUTQVIST, 2001). Os subtipos histológicos de metástases nesta topografia são por ordem de frequência: adenocarcinoma de mama, adenocarcinoma pulmonar, adenocarcinoma prostático, carcinoma de células claras dos rins e os linfomas (ONIMUS et al., 1996; ABDU; PROVENCHER, 1998; SOLBERG; BREMMES, 1999). Os tumores metastáticos que acometem a coluna vertebral são vinte vezes mais frequentes do que os próprios tumores vertebrais primários (PERRIN; LAXTON, 2004).

As metástases vertebrais afetam de 20 a 50\% dos pacientes com câncer e representam uma mudança brusca na qualidade de vida destes indivíduos devido o impacto funcional que geram (ZAIRI; MARINHO; ALLAOUI; ASSAKER, 2012). Elas podem se desenvolver em qualquer faixa etária, sendo que a maior incidência encontra-se entre a $5^{\mathrm{a}}$ e $7^{\mathrm{a}}$ décadas de vida fato relacionado ao risco aumentado de câncer nesta fase da vida. Há uma predominância no sexo masculino decorrente possivelmente ao alto índice de câncer de próstata na população mundial, superando em números absolutos o câncer de mama que é o mais incidente no sexo feminino (PERRIN; LAXTON, 2004; HAREL; ANGELOV, 2010).

A via hematogênica é o caminho mais comum de disseminação metastática para a coluna vertebral, podendo ocorrer por via venosa ou arterial. Na disseminação venosa, o plexo de Batson assume um papel primordial. Ele é composto por veias que não possuem válvulas e que cursam paralelamente e justapostas à coluna vertebral. Este plexo se comunica com vários sistemas venosos (vertebral, cava, porta, ázigos, intercostais, pulmonar, renal) e a direção do fluxo pode ser variável devido às mudanças que ocorrem na pressão intratorácica e intraabdominal. Desta forma, tumores de diversos sítios primários alojados nas cavidades corporais podem depositar células tumorais na coluna vertebral (ROSS et al., 2005). Do mesmo modo, o sistema arterial é capaz de transferir células tumorais para a coluna vertebral devido à boa perfusão que esta região possui (ARGUELLO et al., 1990). 
Um segundo grupo de disseminação é o da extensão direta. Os tumores primários localizados nos tecidos moles paravertebrais podem se estender para a coluna vertebral. O subtipo mais comum a promover este tipo de disseminação é o câncer de pulmão localmente avançado que pode invadir a coluna torácica. Outros tumores como o de próstata, bexiga e cólon podem invadir a coluna lombo-sacra (ROSS et al., 2005).

Outra possível via de disseminação é através do fluido cerebrospinal. Esta propagação acontece pelo derramamento de células tumorais provenientes de lesões metastáticas do cérebro ou cerebelo, muitas vezes após a manipulação cirúrgica de tais metástases cerebrais (PERRIN; LIVINGSTON; AARABI, 1982).

As metástases vertebrais são classificadas em três categorias de acordo com a localização anatômica: extradural, intramedular e intradural-extramedular (KHANNA et al., 2005). As lesões no compartimento extradural são as de maior prevalência, acometendo 9498\% dos casos. Os locais mais acometidos em ordem decrescente são: o corpo vertebral, com ou sem envolvimento do corpo posterior, a região paravertebral e o espaço epidural. As lesões intramedulares e intradural-extramedulares são metástases raras, representando 0,5-1\% dos casos, sendo na maioria das vezes uma propagação intradural da metástase cerebral anterior (SCHICK; MARQUARDT, 2001; PERRIN; LIVINGSTON; AARABI, 1982).

Dentre os pacientes que desenvolverão metástases vertebrais durante a evolução da neoplasia, 10\% apresentarão sintomas ou sequelas (MACCAURO et al., 2011; WITHAM et al., 2006). Uma das queixas de maior prevalência é a compressão vertebral, considerada uma emergência oncológica e que pode comprometer a qualidade de vida do paciente (PUTZ et al., 2008). Ocorre com maior frequência na coluna torácica (60\%), seguida pela coluna lombosacra (25\%) e coluna cervical (10\%). Estas porcentagens estão relacionadas à distribuição relativa do fluxo sanguíneo e à presença de massa óssea em cada segmento vertebral (MEYER; SINGH; JENKINS, 2010; RUFF; RUFF; WANG, 2007). O acometimento de mais de um segmento vertebral é observado em 50\% dos casos (FALAVIGNA et al., 2007; JOAQUIM et al., 2007).

A destruição vertebral causada pelo tumor gera instabilidade óssea e compressão medular, resultando em sintomas graves como dor intratável, disfunção neurológica e incapacidade de deambulação (POINTILLART et al., 2011).

A dor é o primeiro sintoma em 96\% dos pacientes com compressão medular por neoplasia, seguido pelas alterações motoras, sensitivas e autonômicas (QUINN; DEANGELIS, 2000; HAREL; ANGELOV, 2010). São três síndromes clássicas de dor que acometem os pacientes com metástase vertebral: dor local, dor mecânica e dor radicular, 
podendo haver uma combinação dos três tipos (SCIUBBA; GOKASLAN, 2006; HAREL; ANGELOV, 2010). A dor local é descrita como uma dor persistente proveniente da região ou segmento vertebral acometido pela lesão metastática. O crescimento do tumor metastático comumente localizado no corpo vertebral posterior leva a um alongamento periosteal e/ou um processo inflamatório local estimulando assim as fibras dolorosas no periósteo vertebral. A dor mecânica é aquela agravada pelo movimento, pelas atividades mais intensas e pela força de sustentação do peso corporal envolvendo o segmento vertebral afetado. As lesões metastáticas levam à destruição do corpo vertebral gerando instabilidade vertebral e consequente tensão nos músculos, tendões, ligamentos e cápsula que são os sintomas clássicos da dor mecânica. A dor radicular pode ocorrer quando há uma compressão metastática vertebral ou um processo inflamatório na origem da raiz nervosa emergente, produzindo forte dor na distribuição do dermátomo da raiz envolvida (SCIUBBA; GOKASLAN, 2006).

A disfunção motora é o segundo sintoma mais comum, variando de 35 a 75\% (VAN DER LINDEN et al., 2005). Os pacientes podem apresentar alterações mielopáticas e radiculares ou uma combinação das duas. Além disso, podem ter algum nível de disfunção vesical, intestinal e sexual relacionado à compressão medular epidural. Os distúrbios sensoriais como anestesia, hiperestesia e/ou parestesia podem estar presentes e serem relacionados à disfunção motora (SCIUBBA; GOKASLAN, 2006). A queixa mais comum é o peso nas extremidades, que se confirma no exame físico com o déficit motor positivo. Déficits sensoriais podem acompanhar os motores e o controle esfincteriano pode estar preservado no estágio inicial da doença (WITHAM et al., 2006; COLE; PATCHELL, 2008). A disfunção vesical é o sintoma autonômico mais comum e está relacionado com o grau de disfunção motora (SCHIFF, 2003).

\subsection{Diagnóstico e tratamento}

Na última década houve um avanço substancial no diagnóstico e conduta de lesões primárias e metastáticas vertebrais, com consequente aumento da sobrevida destes indivíduos (BARTELS; VAN DER LINDEM; VAN DER GRAAF, 2008; JEMAL et al., 2009).

Para a identificação das metástases ósseas existem várias modalidades de métodos de imagem de diferentes sensibilidades. A radiografia simples é utilizada para rastreio inicial, 
pois é um método barato e está sempre disponível. A tomografia computadorizada produz uma imagem de alta resolução, bastante útil na avaliação dos elementos ósseos da coluna vertebral, de alterações osteolíticas e escleróticas e de colapsos vertebrais. É útil também no planejamento cirúrgico e muitas vezes complementa informações fornecidas pela ressonância magnética para avaliar as alterações ósseas causadas pelo tumor. A principal desvantagem é a baixa acurácia para diferenciar as estruturas do tecido mole e a presença de envolvimento ósseo (JACOBS; PERRIN, 2001; GUILLEVIN et al., 2007). A cintilografia óssea é rotineiramente usada para triagem do envolvimento ósseo na oncologia e possui sensibilidade de 62 a 89\%, sobretudo para lesões osteoblásticas (DALDRUP-LINK et al., 2001; COSTELLOE et al., 2009).

A ressonância magnética é uma modalidade de imagem de alta resolução, padrão ouro para avaliar doença metastática vertebral. Ela permite identificar todos os aspectos da coluna vertebral, diferenciando a estrutura óssea dos tecidos moles ao redor, permitindo assim a visualização da medula espinhal, raízes nervosas, meninges, espaço intervertebral e musculatura paravertebral. Este exame é mais sensível para detectar tumores ósseos malignos primários e metastáticos na coluna vertebral quando comparado às radiografias convencionais, à tomografia computadorizada e à cintilografia óssea (ANDREULA; MURRONE, 2005; JUNG et al., 2003).

O tratamento da metástase óssea vertebral é primariamente paliativo na perspectiva oncológica, pois tem como objetivo o alívio da dor, a preservação da função neurológica e a manutenção ou recuperação da estabilidade vertebral. Algumas variáveis como idade, carga tumoral, expectativa de vida e capacidade funcional influenciarão na escolha das opções terapêuticas (SCIUBBA; GOKASLAN, 2006; JU et al., 2013).

Duas abordagens terapêuticas têm sido eficazes no tratamento da doença metastática vertebral: a radioterapia e a cirurgia. Estudos nas décadas de 60 e 70 não demonstraram diferença nos resultados entre os pacientes tratados somente com radioterapia e aqueles tratados com a descompressão cirúrgica com ou sem radioterapia (YOUNG; POST; KING, 1980). O tratamento cirúrgico nestes estudos consistia em laminectomia (retirada da lamina dorsal vertebral), o que atualmente é considerado inadequado, pois desestabiliza ainda mais a coluna vertebral (SCIUBBA et al., 2010).

Os avanços nas técnicas cirúrgicas e nos instrumentais utilizados têm resultado em cirurgias mais eficazes na descompressão medular. Estas novas técnicas permitem a reconstrução imediata e estabilização da coluna vertebral, mesmo em pacientes com destruição óssea e comprometimento medular causado pelas metástases (FALICOV et al., 
2006; JANSSON; BAUER, 2006). Os benefícios trazidos pela descompressão posterior que aborda a ressecção tumoral e a estabilização da coluna vertebral supera a instabilidade iatrogênica decorrente da laminectomia (VRIONIS; SMALL, 2003).

A cifoplastia e a vertebroplastia são procedimentos cirúrgicos minimamente invasivos realizados na coluna lombar e/ou torácica, indicados em pacientes com dor axial secundária ao colapso do corpo vertebral e que não possuem evidência de compressão de estruturas nervosas. A vertebroplastia consiste na injeção percutânea, normalmente através do pedículo vertebral, de metil metacrilato (cimento ósseo) no interior do corpo vertebral. Na cifoplastia, diferentemente da vertebroplastia, realiza-se dilatação prévia do corpo vertebral colapsado através de balão apropriado com formação de uma cavidade, permitindo injeção da referida substância com pressão gradual. Ambos os procedimentos são realizados sob a orientação de radioscopia ou tomografia computadorizada e objetivam o preenchimento de dois terços anteriores do corpo com metil metacrilato e consequente correção da deformidade (VACCARO; BETZ; ZEIDMAN, 2003; DANIEL; VEIGA, 2007; QURAISHI; GOKASLAN; BORIANI, 2010; SCIUBBA et al.; 2010). As principais complicações destes métodos são o extravasamento inadvertido de metil metacrilato para o interior do canal vertebral com consequente lesão de estruturas nervosas e a absorção pelas veias do plexo epidural desta substância produzindo embolia pulmonar (DANIEL; VEIGA, 2007). O controle da dor é possível em 50-80\% dos casos (HEARY; BONO, 2001).

Nos pacientes com compressão significativa de elementos nervosos associados à instabilidade significativa da coluna vertebral, faz-se necessário a descompressão da medula espinhal, reconstrução do corpo vertebral e estabilização da coluna vertebral, visando à manutenção das propriedades anatômicas e biomecânicas da coluna e preservação da função neurológica (HEARY; BONO, 2001; VACCARO; BETZ; ZEIDMAN, 2003; DANIEL; VEIGA, 2007; QURAISHI; GOKASLAN; BORIANI, 2010 ).

$\mathrm{O}$ acesso cirúrgico à coluna vertebral pode ser realizado por via anterior, via posterior ou combinada. A via anterior tem como objetivo o acesso ao corpo vertebral, que pode ser realizado através de cervicotomia anterior, toracotomia ou toracoscopia, laparotomia retroperitoneal ou laparoscopia na dependência do segmento vertebral acometido. Estas vias são utilizadas nos casos com indicação de descompressão anterior através de corpectomia ou vertebrectomia. Após a descompressão, é fundamental a reconstrução do corpo com implantes de titânio (cage, placas e parafusos). A via posterior é a mais utilizada na prática neurocirúrgica e baseia-se na laminectomia seguida de fixação através de parafusos transpediculares de titânio das vértebras adjacentes. A extensão da fixação dependerá da 
qualidade óssea, do segmento vertebral acometido, do tipo de implante e da presença de metástases em outras vértebras (HEARY; BONO, 2001; VACCARO; BETZ; ZEIDMAN, 2003; QURAISHI; GOKASLAN; BORIANI, 2010).

Existem poucos estudos prospectivos sobre as indicações de tratamento e resultados cirúrgicos em pacientes com doença metastática vertebral. A literatura, mesmo se tratando de dados retrospectivos, sugere que em pacientes bem selecionados, a cirurgia pode proporcionar uma melhora significativa na qualidadede vida em geral (HESSLER et al., 2009; VRIONIS; SMALL, 2003).

O estudo de Patchell et al. (2005) tornou-se o ponto de referência na indicação e escolha do procedimento cirúrgico a ser realizado, demonstrando excelentes benefícios para os indivíduos selecionados. Cirurgias para descompressão medular, embora permaneçam paliativas, tornaram-se o tratamento padrão para lesões metastáticas. Novas técnicas em combinação com métodos de imagem que detectam doença vertebral em estágios iniciais têm demonstrado melhores resultados (WITHAM et al., 2006).

As indicações do tratamento cirúrgico em pacientes com metástases vertebral baseiamse em uma série de fatores: evidência de progressão tumoral após realização de radioterapia prévia, piora do quadro neurológico durante radioterapia, compressão medular significativa, dor intratável, metástase solitária quando ambos os tumores primário e espinhal são passíveis de cirurgia, ausência de diagnóstico, tumor radio-resistente e evidência de instabilidade vertebral (CHAICHANA et al., 2009).

\subsection{Qualidade de vida relacionada à saúde (QVRS) e o papel da fisioterapia}

A Organização Mundial da Saúde (OMS) define Qualidade de Vida (QV) como a percepção do indivíduo e da sua posição na vida, no contexto cultural e nos sistemas de valores nos quais vive e em relação aos seus objetivos, expectativas, padrões e preocupações (WHO, 1993).

A avaliação da QV em situações de saúde é denominada Qualidade de Vida Relacionada à Saúde (QVRS). Segundo Fayers e Machin (2007), QVRS é usado como o estado de saúde percebido e tem como objetivo principal verificar o quanto a doença ou o estado crônico, além dos sintomas, passam a interferir na vida diária do indivíduo. 
A mensuração da QVRS do paciente oncológico é um importante recurso para avaliar os resultados do tratamento na perspectiva do paciente e o impacto do mesmo no seu cotidiano. Uma avaliação multidimensional da QVRS é relevante para os indivíduos submetidos à cirurgia paliativa. Domínios psicológicos, sociais e físicos são medidas de resultados importantes nesta população (CELLA, 1996).

Como referencial teórico, utilizamos nesta pesquisa o modelo de QVRS para sobreviventes de câncer elaborado por Ferrel, Dow e Grant (1995). Este modelo centraliza os domínios de bem-estar físico, social, psicológico e espiritual, correlacionando-os de forma dinâmica, com influência na QVRS do paciente. Caso ocorra alguma alteração em um desses domínios, a QVRS será afetada como um todo. Um sintoma físico não controlado compromete o bem-estar psicológico, gerando ansiedade, depressão e frustração ao paciente, colocando em risco a dimensão social do mesmo. Algumas limitações do bem-estar físico vivenciadas por ele podem restringir seu convívio social, exigindo mais da família e do cuidador para desenvolver suas atividades que estão limitadas.

Segundo estes mesmos autores, o bem-estar físico é definido como o controle ou o alívio dos sintomas e a manutenção da função e da independência. O bem-estar social é uma forma de visualizar não apenas o câncer com os seus sintomas, mas também o significado do impacto da doença e do tratamento no cotidiano da pessoa com câncer, provocando a perda de seus papéis e relacionamentos. O bem-estar psicológico busca um equilíbrio diante das ameaças da vida causadas pela doença. Estas ameaças levam ao estresse emocional que modifica as prioridades da vida e gera o medo do desconhecido. O bem-estar espiritual é caracterizado pelas dimensões existenciais e religiosas. Ele é pautado pelos sentimentos de incertezas, desesperanças, mudanças espirituais e aumento do significado da vida.

O domínio funcionalidade que tem como definição a capacidade percebida pelo indivíduo em executar suas funções e atividades da vida diária em relação às expectativas e adaptações ao declínio da funcionalidade, será avaliado neste estudo para evidenciar as mudanças no pós-operatório quando comparadas à fase pré-operatória.

Quando falamos em QVRS do paciente, devemos buscar um envolvimento harmonioso de toda a equipe de saúde, do paciente e da família para uma melhor comunicação, compreensão e suporte durante todo o processo saúde-doença. Além disso, o fisioterapeuta como integrante da equipe multiprofissional e pela sua proximidade com os pacientes ao longo do tratamento oncológico deve estar preparado e capacitado para apoiar o paciente em todas as situações de dificuldade, sejam elas física, emocional, social, cultural 
e/ou espiritual. Além disso, avaliar a QVRS permite levantar os domínios afetados e auxilia no planejamento da assistência fisioterapêutica para a reabilitação desses pacientes.

A confederação mundial de fisioterapia (World Confederation for Physical Therapy WCPT) define esta especialidade como prestadora de serviços a todos os indivíduos com o objetivo de desenvolver, manter ou restaurar o movimento máximo e a capacidade funcional ao longo da vida. O fisioterapeuta entra em cena quando a funcionalidade dos movimentos do corpo está ameaçada em virtude de algum processo patológico (WCPT, 2007). Sendo assim, no âmbito oncológico, sua atuação é interdisciplinar na promoção à saúde, diagnóstico precoce e tratamento dos distúrbios cinéticos funcionais provenientes das intercorrências oncológicas, resgatando a funcionalidade do indivíduo, por meio do diagnóstico fisioterapêutico, prescrição e execução de métodos, técnicas e recursos terapêuticos e educativos.

A fisioterapia possui um arsenal abrangente de técnicas e recursos que complementam os cuidados em cada fase da doença, tanto na melhora da sintomatologia quanto na qualidade de vida. Entre as principais indicações estão o alívio da dor, a melhora das complicações osteomioarticulares e o controle da fadiga.

Quando falamos em alívio da dor devemos lembrar que este sintoma é constituído por componentes físicos, mentais, sociais e espirituais. Dentre as intervenções fisioterapêuticas para a dor temos a eletroterapia que traz resultados rápidos, porém com alívio variável entre os pacientes. No contexto terapêutico atual, não é possível tratar a dor oncológica somente com o uso de corrente elétrica analgésica, mas é possível diminuir de forma significativa o uso de analgésicos e consequentemente seus efeitos colaterais (IAHPC, 2003). Os métodos de terapia manual também podem ser utilizados para complementar o alívio da dor, diminuindo a tensão muscular, melhorando a circulação tecidual e diminuindo a ansiedade do paciente (MAIN; WATSON, 1999; PIMENTA, 2003). Também para diminuição da tensão muscular gerada pela dor, o uso de alongamentos é eficaz e pode ser utilizado com relativa facilidade e baixo custo, sempre que possível com orientação de um fisioterapeuta.

As complicações osteoarticulares são bastante frequentes nesta população, sendo denominada Síndrome do Desuso. É caracterizada pelo excesso de descanso e inatividade física, o que pode gerar ou agravar o estado de dor, dentre outras complicações. Esta síndrome é composta por fraqueza muscular (hipotrofia), descondicionamento cardiovascular, respiração superficial e alterações posturais. O retorno à atividade física gera um processo de regeneração, aumentando a síntese proteica e o realinhamento das fibras musculares (SODERBERG, 1997; PIMENTA, 2003). 
A fadiga é um fenômeno complexo, composto por uma percepção subjetiva de cansaço, por alterações do tecido neuromuscular e dos processos metabólicos, pela diminuição da performance física, da motivação e das atividades mentais. As intervenções para a fadiga têm sido sugeridas em dois níveis: o manejo dos sintomas que contribuem para a fadiga e a prevenção da fadiga, mantendo o equilíbrio do período de descanso com o de atividade (FERREL; COYLE, 2001).

O fisioterapeuta como membro da equipe de saúde atua de forma complementar a fim de obter, dentro de seu alcance profissional, o cuidado que o paciente necessita, delineando sua função e investigando quais os melhores recursos fisioterapêuticos a serem utilizados. $\mathrm{O}$ benefício a ser buscado é preservar a vida e aliviar os sintomas, dando oportunidade, sempre que possível, para a independência funcional do paciente e prestar orientações ao cuidador sobre o manuseio do paciente no domicilio.

Sabemos que a doença metastática leva a uma esperança de vida limitada, em que a capacidade funcional e a QVRS são objetivos extremamente importantes de serem alcançados pelo paciente e pelos seus familiares. Certamente alguns fatores físicos como deambulação, redução da dor e aumento da sobrevida tornam-se itens importantes para uma boa QVRS (FUJIBAYASHI et al., 2010).

Dentro deste contexto vale destacar a importância da abordagem multidisciplinar nos cuidados prestados no âmbito oncológico, pois implica em demonstrar que nenhuma profissão consegue abranger todos os aspectos envolvidos no tratamento de indivíduos sem possibilidade de cura, o que faz destacar a significância do trabalho coletivo, permitindo a sinergia de habilidades para promover uma assistência completa (MCCOUGHLAN, 2003).

Poucos estudos prospectivos avaliaram pacientes com metástases vertebrais submetidos a procedimentos cirúrgicos de descompressão medular e sua Qualidade de Vida Relacionada à Saúde devido às dificuldades de se pesquisar esta população. Outras adversidades encontradas foram: estudos que tinham como propósito avaliar a QVRS, mas só traziam em seus resultados um enfoque nas escalas funcionais e não na qualidade de vida global; tentativas de estudo com análises mais sofisticadas, prejudicados pelo tamanho da amostra; elevado número de pacientes perdidos no follow-up; períodos de acompanhamento curto (WEIGEL et al., 1999; WAI et al., 2003; WANG et al., 2004)

No estudo de Falicov et al. (2006), foram avaliados prospectivamente 85 pacientes com metástase vertebral submetidos à cirurgia para descompressão medular com o objetivo de analisar a Qualidade de Vida Relacionada à Saúde após o procedimento. O autor utilizou o instrumento da European Organization for Research and Treatment of Cancer Quality of 
Life Questionnaire "core" 30 item (EORTC QLQ-C30) e a escala de capacidade funcional ECOG Performance Status (Eastern Cooperative Oncology Group) antes da intervenção cirúrgica, e após seis semanas, três e seis meses e um ano. O domínio Estado de Saúde Global/Qualidade de Vida (ESG/QV) do instrumento EORTC QLQ C-30 apresentou resultado estatisticamente significativo quando se comparou o período pré-operatório com seis semanas $(p=0,0017)$, com três meses $(p=0,039)$ e com seis meses após o procedimento cirúrgico $(\mathrm{p}=0,013)$. Além disso, o item Dor da Escala de Sintomas do mesmo instrumento também mostrou resultados estatisticamente significativos, incluindo o período de um ano após a cirurgia, sempre comparando com o estado pré-operatório - seis semanas $(\mathrm{p}<0,0001)$, três meses $(p=0,0015)$, seis meses $(p=0,0016)$ e um ano $(p=0,0017)$. Não houve diferença estatisticamente significativa para a capacidade funcional dos participantes. Com os resultados desse estudo, comprovou-se que a intervenção cirúrgica em casos de lesão metastática vertebral compressiva oferece aos pacientes benefícios, como melhora dos sintomas e da Qualidade de Vida, com baixo índice de complicação cirúrgica.

Diante do exposto acima, o presente estudo apresenta relevância científica devido à escassez de dados, sobretudo na literatura brasileira, para comparar cirurgia de descompressão medular decorrente de metástases ósseas na coluna vertebral e a melhora da Qualidade de Vida Relacionada à Saúde (QVRS) após a realização deste procedimento. Através da correlação dos resultados apresentados neste trabalho, poderá ocorrer um melhor planejamento da assistência multiprofissional a estes pacientes. 


\section{OBJETIVOS}

\section{Objetivo geral:}

- Avaliar a Qualidade de Vida Relacionada à Saúde (QVRS) e a capacidade funcional dos indivíduos com metástase na coluna vertebral antes e após serem submetidos ao tratamento cirúrgico de descompressão medular.

\section{Objetivos específicos:}

- Caracterizar o perfil (sociodemográfico e clínico) dos pacientes com metástase vertebral que serão submetidos à cirurgia de descompressão medular;

- Descrever os domínios de QVRS do instrumento The European Organization for Research and Treatment of Cancer (EORTC) QLQ-C30 (Quality of life Questionnaire - Core 30) nos quatro momentos avaliados: pré-operatório (T0), dois meses após a cirurgia (T1), quatro meses após a cirurgia (T2) e seis meses após a cirurgia (T3).

- Comparar os domínios de QVRS no pré-operatório (T0) com o T1, T2 e T3.

- Comparar o domínio dor do instrumento EORTCQLQ-C30 com a Escala de Dor de Faces Revisada (FPS-R) nos quatro momentos avaliados;

- Comparar a capacidade funcional nos quatro momentos avaliados no estudo. 
MATERIAIS E MÉTODOS 


\section{MATERIAIS E MÉTODOS}

\subsection{Delineamento do estudo}

Trata-se de um estudo quantitativo, descritivo, correlacional e de corte longitudinal do tipo prospectivo que englobará quatro avaliações: antes da cirurgia, dois, quatro e seis meses após o procedimento cirúrgico (POLIT; BECK, 2011).

\subsection{Local}

O estudo foi realizado nas unidades de internação e ambulatorial do Serviço de Coluna pertencente ao Departamento de Biomecânica, Medicina e Reabilitação do Aparelho Locomotor. O serviço atende portadores de afecções ósseas de ambos os sexos, sem limite de idade, das mais variadas regiões da cidade e do país, pelo Sistema Único de Saúde (SUS) e por meio de convênios nas dependências do Hospital das Clínicas da Faculdade de Medicina de Ribeirão Preto da Universidade de São Paulo (HC-FMRP). Essa instituição é considerada referência na área da saúde, tecnologia e atendimento de casos de alta complexidade.

\subsection{População e amostra}

Os participantes em potencial deste estudo foram pacientes oncológicos que estavam em tratamento no local selecionado descrito anteriormente.

Para a seleção dos participantes foram adotados os seguintes critérios de inclusão: ter indicação de tratamento cirúrgico da coluna vertebral devido compressão medular causada por metástase óssea, apresentar-se consciente, lúcido e com capacidade de verbalização para responder aos itens dos instrumentos, avaliados por perguntas simples como qual o dia da semana, data de nascimento e outros, e ter idade igual ou superior a 18 anos de idade. 
Não participaram do estudo os pacientes que apresentavam o diagnóstico de depressão grave informada no prontuário médico visto que o aspecto cognitivo era primordial para que o indivíduo pudesse responder às solicitações necessárias no momento da entrevista e na avaliação da sua qualidade de vida relacionada à saúde.

Também não participaram do estudo pacientes que apresentavam diagnóstico de compressão medular decorrente de um tumor primário da coluna vertebral como, por exemplo, mieloma múltiplo ou osteossarcoma.

A amostra deste estudo foi formada por 22 pacientes com tumor metastático para a coluna vertebral, que foram submetidos à cirurgia em decorrência do diagnóstico de compressão medular neoplásica metastática.

\subsection{Aspectos Éticos da Pesquisa}

Este estudo foi submetido e aprovado pelo Comitê de Ética em Pesquisa (CEP) da Escola de Enfermagem de Ribeirão Preto da Universidade de São Paulo (USP) em reunião de 16 de outubro de 2013 com o numero de protocolo 10801713.2.0000.5393 (Anexo 5).

Para cada sujeito da pesquisa, antes do início da coleta de dados, foi entregue um “Termo de Consentimento Livre e Esclarecido” (TCLE) (Apêndice 1) de acordo com Resolução do Conselho Nacional de Saúde (CNS) 466/12. Todos os participantes tiveram que assinar o referido termo confirmando o aceite para participar do estudo.

\subsection{Coleta de Dados}

Os dados foram coletados por entrevistas realizadas pela pesquisadora, nas unidades de internação (fase pré-operatória) e no ambulatório do Serviço de Coluna pertencente ao Departamento de Biomecânica, Medicina e Reabilitação do Aparelho Locomotor do HCFMRP (aos dois, quatro e seis meses após a data da cirurgia). As entrevistas foram realizadas no período de outubro de 2013 a agosto de 2014 (primeira avaliação). O término da avaliação de seis meses após a cirurgia do último participante do estudo foi encerrado em fevereiro de 2015. 
Para a realização das entrevistas, a pesquisadora era informada pelo médico residente do Serviço de Coluna pertencente ao Departamento de Biomecânica, Medicina e Reabilitação do Aparelho Locomotor da data e do horário do procedimento cirúrgico. Após a indicação pelo médico, realizava-se a verificação no prontuário para certificação do diagnóstico e obtenção de informações gerais sobre o paciente. Em seguida, era feito contato com o paciente na enfermaria e auto apresentação da pesquisadora e dos objetivos da pesquisa. $\mathrm{Na}$ sequência, era formalizado o convite para participar do estudo com a apresentação do TCLE, fornecendo todas as informações necessárias e requeridas pelo paciente e acompanhante. Quando o paciente encontrava-se impossibilitado por condições físicas de assinar o TCLE, a assinatura deste documento era solicitada ao acompanhante.

Após a assinatura do termo, iniciava-se a entrevista na própria enfermaria à beira do leito, com duração de aproximadamente 30 minutos. Ao término da entrevista, a pesquisadora apresentava os agradecimentos aos participantes e se dispunha para qualquer informação que se fizesse necessária e já informava que as próximas entrevistas seriam realizadas no ambulatório do Serviço de Coluna do HC-FMRP, coincidindo com os retornos médicos aos dois, quatro e seis meses após a cirurgia.

\subsection{Instrumentos de coleta de dados}

\subsubsection{Instrumento para caracterização sociodemográfica e clínica}

Com a finalidade de traçar o perfil sociodemográfico e clínico dos indivíduos incluídos nesta pesquisa, foi elaborado pela pesquisadora um questionário dividido em dados sociodemográficos e dados clínicos pertinentes à doença oncológica (Apêndice 2).

Os dados sociodemográficos incluíam sexo, idade, cor, estado civil, escolaridade, ocupação, situação profissional e renda familiar. Os dados clínicos incluíam localização do tumor primário, nível(eis) da lesão metastática vertebral, tipo de procedimento cirúrgico e tipo de tratamento pós-operatório. 


\subsubsection{Escala de Dor de Faces Revisada (FPS-R)}

Esta escala consistia originalmente de sete faces, apresentadas em escala crescente de intensidade da dor, havendo dificuldade na analogia com a relação métrica ( 0 a 5 ou 0 a 10), uma vez que ela é uma escala de sete pontos. Essa escala era de fácil administração e não requeria equipamento excepcional, exceto as “faces” fotocopiadas (BIERI et al.,1990). Hicks et al (2001) revisaram esta escala original e idealizaram uma escala de auto-relato que consistia em seis faces apresentadas horizontalmente, sem expressõe8s de choros ou sorrisos para expressar diferentes graus de dor desde “sem dor” até “a maior dor possível”, fazendo a correlação métrica de 0-10, onde zero corresponde a sem dor e dez a maior dor possível. É uma escala crescente, e para cada face é atribuído um valor de 0 a 10 (0, 2, 4, 6, 8 e 10) sendo chamada de Escala de Faces Revisada (FPS-R). Esta escala de faces revisada foi traduzida e validada no Brasil por Silva e Thuler (2008) (Anexo1).

\subsubsection{Instrumento de avaliação da qualidade de vida relacionada à saúde - EORTC QLQ-C30}

O instrumento EORTC QLQ-C30 (European Organization for Research and Treatment of Cancer Quality of Life Questionnaire “core” 30 item) (Anexo 2) foi desenvolvido por esta organização europeia de pesquisa e tratamento do câncer com a finalidade de avaliar a Qualidade de Vida de pacientes com câncer de pulmão (AARONSON et al., 1993). A partir deste estudo, esta escala ficou universalmente reconhecida como válida e confiável para avaliar Qualidade de Vida em uma população com câncer.

Até o momento, este instrumento foi validado e traduzido em 81 idiomas, inclusive na língua portuguesa (Brasil), com ampla utilização clínica e em pesquisas no mundo todo. No Brasil, foi validado em 2006 por Brabo.

O instrumento EORTC QLQ-C30 versão 3.0 é um questionário composto por 16 domínios, 30 questões, incorporando quatro escalas (Quadro 1) (FAYERS et al., 2001):

$\checkmark$ Escala de Estado de Saúde Global e Qualidade de Vida (ESG/QV), contendo um domínio e dois itens;

$\checkmark$ Escala Funcional, contendo cinco domínios e 15 itens; 
$\checkmark$ Escala de Sintomas, contendo nove domínios e 12 itens;

$\checkmark$ Escala de Dificuldade Financeira, contendo um domínio e um item.

Quadro 1 - Escores do EORTC QLQ-C30 Versão 3.0

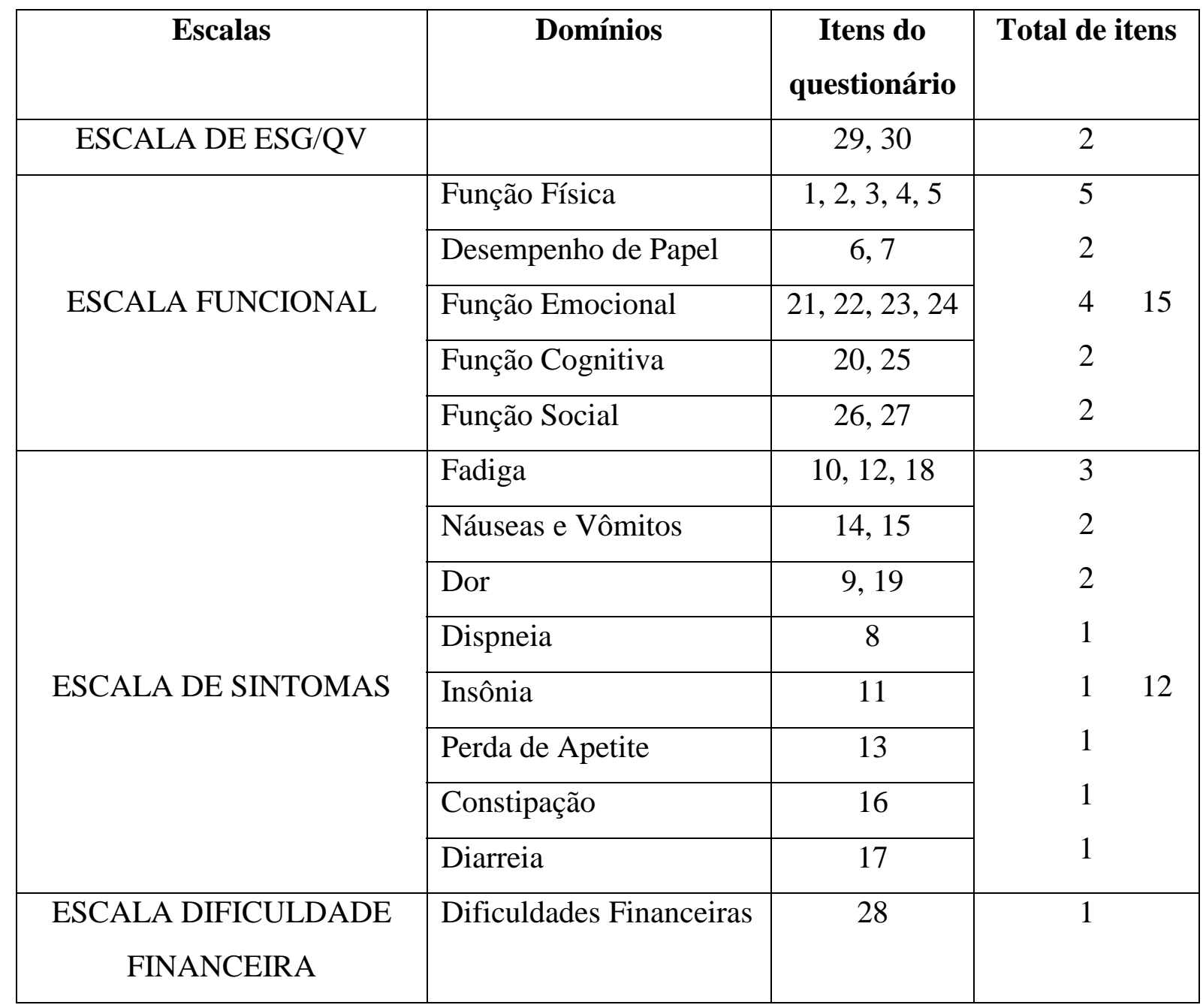

(Adaptado de Fayers et al., 2001)

As questões de 01 a 28 estão dispostas em escala tipo Likert de quatro pontos, com respostas variando de não (valor do escore $=1$ ) a muito (valor do escore $=4$ ) $($ Figura 1$)$. As questões 29 e 30, também dispostas em escala do tipo Likert, apresentam sete pontos, com assertivas desde péssimo (escore $=1$ ) a ótimo (escore $=7$ ) (Figura 2).

1

Não
2

Pouco

3

Moderadamente

4

Muito

Figura 1 - Escala de Likert de quatro pontos - EORTC QLQ-C30 


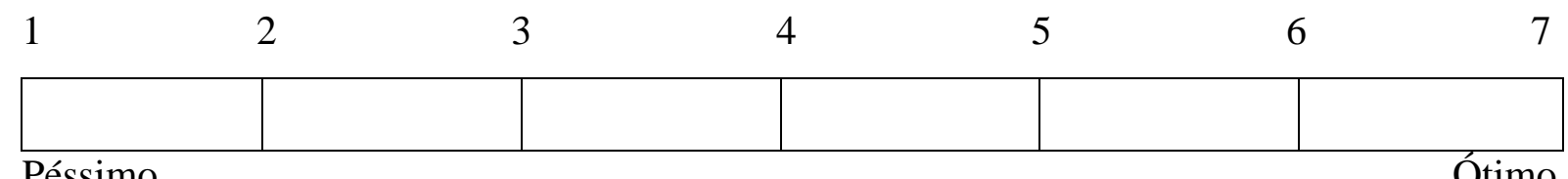

Figura 2 - Escala de Likert de sete pontos - EORTC QLQ-C30

O escore de medição dos itens varia com uma pontuação de 0 a 100 . A pontuação elevada para a escala funcional representa um nível saudável de funcionalidade. A pontuação alta para o ESG/QV representa uma boa Qualidade de Vida. No entanto, uma pontuação elevada para escala de sintomas representa um elevado nível de sintomatologia e uma pior Qualidade de Vida (FAYERS et al., 2001).

Para a utilização deste instrumento, foi realizado o registro da pesquisa no site http://groups.eortc.be/qol/questionnaire_qlqc30.htm, onde foi solicitada a permissão de uso nesta pesquisa, obtendo-se a autorização (Anexo 3).

\subsubsection{Instrumento para avaliar a capacidade funcional - Índice de Barthel}

O Índice de Barthel (MAHONY; BARTHEL, 1965) (Anexo 4) pertence ao campo de avaliação das Atividades de Vida Diária (AVDs) e mede a independência funcional no cuidado pessoal, mobilidade, locomoção e hábitos fisiológicos. Utilizamos a versão traduzida e adaptada por Minosso et al. (2010) em que cada item é pontuado de acordo com o desempenho do indivíduo em realizar tarefas de forma independente, com alguma ajuda ou de forma dependente. Este instrumento avalia a independência funcional em dez tarefas: alimentação, banho, vestuário, higiene pessoal, eliminações intestinais, eliminações vesicais, uso do banheiro, passagem da cadeira para a cama e vice-versa, deambulação e subir e descer escadas. Uma pontuação geral é atingida, atribuindo-se pontos em intervalos de cinco para cada categoria, na dependência das necessidades de cada paciente. Dois itens (banho e higiene pessoal) apresentam escala de duas alternativas (zero e cinco pontos); seis itens (alimentação, vestuário, eliminações vesical e intestinal, uso do banheiro e subir e descer escadas) apresentam escala de três alternativas (zero, cinco e dez pontos); dois itens restantes (transferência e deambulação) apresentam escala de quatro alternativas (zero, cinco, dez e quinze pontos). A pontuação final é a soma dos pontos de cada item, variando de zero (completa dependência) a 100 (independente). 


\section{PROCESSAMENTO E ANÁLISE DOS DADOS}

Os dados foram inseridos em uma planilha do programa Microsoft Office Excel for Windows 2007, submetidos à avaliação de consistência de dupla digitação e posteriormente transportados para a análise estatística no programa Statistical Package for the Social Sciences (SPSS) versão 16.0. Foram realizadas análises descritivas de frequência simples para as variáveis categóricas (sexo, cor, estado civil, nível de escolaridade, ocupação, situação ocupacional, renda mensal, diagnóstico primário, tipo de procedimento cirúrgico, nível da lesão metastática e tratamento pós-operatório) e de medidas de tendência central (mediana e média) e de variabilidade (desvio-padrão (DP)) para as variáveis contínuas idade e para as medidas de QVRS.

Para comparar os escores que avaliam a QVRS e a capacidade funcional apresentados no pré-operatório (T0) e os resultados percebidos pelos pacientes aos dois (T1), quatro (T2) e seis meses (T3) após a cirurgia, foram utilizados o Teste t de Student pareado. Para comparar as medidas dos domínios de QVRS, dor (escala FPS-R) e da limitação funcional percebida antes e depois do tratamento cirúrgico, utilizamos o Teste t de Student pareado.

Todos os testes estatísticos foram realizados admitindo um nível de significância de 0,05 . 
RESULTADOS 


\section{RESULTADOS}

\subsection{Caracterização sociodemográfica dos pacientes com metástase vertebral}

Participaram do estudo 22 indivíduos com doença metastática na coluna vertebral com indicação cirúrgica para alívio dos sintomas decorrente da compressão medular. Todos os indivíduos eram acompanhados no Serviço de Coluna do Departamento de Biomecânica, Medicina e Reabilitação do Aparelho Locomotor e foram incluídos no período de outubro de 2013 a agosto de 2014. Neste mesmo período, outros quatro pacientes apresentaram indicação de cirurgia para descompressão medular. Eles não foram incluídos para análise estatística, pois tinham como diagnóstico mieloma múltiplo ou tumores primários da coluna vertebral, não preenchendo critérios para inclusão no estudo.

Em relação às características sociodemográficas dos participantes (Tabela 1), 11 (50\%) eram do sexo feminino e 11 (50\%) do sexo masculino. A idade variou de 33 a 87 anos, com uma mediana de 57,00 (DP = 13,50); na faixa etária acima dos 60 anos, que caracteriza a população de idosos segundo definição da Organização Mundial de Saúde (OMS), estavam 45\% dos pacientes. Dos 22 pacientes incluídos, 17 (77,3\%) viviam com um(a) companheiro(a).

Levando-se em conta o nível de escolaridade dos entrevistados, 15 (68,2\%) pacientes possuíam o nível de ensino fundamental, cinco (22,7\%) o nível de ensino médio e apenas dois $(9,1 \%)$ haviam concluído o ensino superior. Quanto à situação ocupacional, nenhum dos participantes do estudo estava trabalhando no momento da coleta, sendo que 11 (50\%) declararam estar aposentados(as) e 11 (50\%) afastados do trabalho. As ocupações prévias de cada um dos sujeitos de pesquisa eram: cinco (22,7\%) do lar, um (4,5\%) cabeleireiro(a), um $(4,5 \%)$ tratorista, três $(13,6 \%)$ motorista, um $(4,5 \%)$ policial civil, dois $(9,1 \%)$ doméstica, um (4,5\%) servente de pedreiro, um(a) (4,5\%) autônomo(a), um (4,5\%) operador de bomba de água, dois $(9,1 \%)$ comerciante, um $(4,5 \%)$ conferente de carga, um $(4,5 \%)$ calheiro, um $(4,5 \%)$ costureiro(a) e um $(4,5 \%)$ pedreiro.

A renda mensal média de cada um dos participantes ficou assim dividida: 13 (59,1\%) recebiam um salário mínimo, quatro (18,2\%) recebiam dois salários mínimos, dois $(9,1 \%)$ recebiam até três salários mínimos e três $(13,6 \%)$ recebiam quatro ou mais salários mínimos. 
Tabela 1 - Frequência e porcentagem das características sociodemográficas dos pacientes $(n=22)$ com metástase vertebral do Hospital das Clínicas da Faculdade de Medicina de Ribeirão Preto - USP, 2013-2015.

\begin{tabular}{|c|c|c|}
\hline Variáveis & $\mathbf{N}$ & $\%$ \\
\hline \multicolumn{3}{|l|}{ Sexo } \\
\hline Masculino & 11 & 50 \\
\hline Feminino & 11 & 50 \\
\hline \multicolumn{3}{|l|}{ Cor } \\
\hline Branca & 14 & 63,6 \\
\hline Negra & 6 & 27,3 \\
\hline Parda & 2 & 9,1 \\
\hline \multicolumn{3}{|l|}{ Faixa etária (anos) } \\
\hline Até 60 & 12 & 54,5 \\
\hline$>60$ & 10 & 45,5 \\
\hline \multicolumn{3}{|l|}{ Estado civil } \\
\hline Com companheiro & 17 & 77,3 \\
\hline Sem companheiro & 5 & 22,7 \\
\hline \multicolumn{3}{|l|}{ Nível escolaridade } \\
\hline Fundamental & 15 & 68,2 \\
\hline Médio & 5 & 22,7 \\
\hline Superior & 2 & 9,1 \\
\hline \multicolumn{3}{|l|}{ Ocupação } \\
\hline Do lar & 5 & 22,7 \\
\hline Motorista & 3 & 13,6 \\
\hline Doméstica & 2 & 9,1 \\
\hline Comerciante & 2 & 9,1 \\
\hline Cabeleireiro & 1 & 4,5 \\
\hline Tratorista & 1 & 4,5 \\
\hline Polícia Civil & 1 & 4,5 \\
\hline Servente de pedreiro & 1 & 4,5 \\
\hline Autônomo & 1 & 4,5 \\
\hline Operador de bomba de água & 1 & 4,5 \\
\hline Conferente de carga & 1 & 4,5 \\
\hline Calheiro & 1 & 4,5 \\
\hline Costureiro & 1 & 4,5 \\
\hline Pedreiro & 1 & 4,5 \\
\hline \multicolumn{3}{|l|}{ Situação ocupacional } \\
\hline Aposentado & 11 & 50 \\
\hline Afastado do trabalho & 11 & 50 \\
\hline \multicolumn{3}{|c|}{ Renda mensal (salário mínimo)* } \\
\hline Um & 13 & 59,1 \\
\hline Dois & 4 & 18,2 \\
\hline Três & 2 & 9,1 \\
\hline Quatro ou mais & 3 & 13,6 \\
\hline
\end{tabular}




\subsection{Caracterização clínica dos pacientes com metástase vertebral}

Com relação à caracterização clínica dos pacientes com metástase vertebral, verificouse que quanto à localização primária do câncer (Tabela 2), quatro sítios representaram mais de 80\% dos casos. A ordem de incidência foi: câncer de mama (40,9\%), câncer de próstata (18,2\%), câncer de pulmão (13,6\%) e câncer colorretal (13,6\%).

Quanto ao procedimento cirúrgico realizado, foi verificado que 63,6\% dos pacientes foram submetidos a uma abordagem vertebral posterior (AVP), 22,7\% submetidos à cifoplastia, 9,1\% a uma abordagem vertebral anterior (AVA) e 4,5\% a uma abordagem anterior e posterior (AVA+AVP).

Com relação ao nível da lesão metastática vertebral, 36,4\% encontravam-se na região lombar, 31,8\% na região torácica, $13,6 \%$ na região cervical, 13,6\% região toracolombar e 4,5\% na região lombo-sacra.

Quanto ao tratamento pós-operatório, constatou-se que 50\% dos pacientes não realizaram nenhum tipo de tratamento complementar, 22,7\% realizaram radioterapia paliativa “adjuvante”, 18,2\% realizaram quimioterapia paliativa e 9,1\% realizaram a associação de quimioterapia e radioterapia.

Tabela 2 - Frequência e porcentagem das características clínicas dos pacientes (n=22) com metástase vertebral do Hospital das Clínicas da Faculdade de Medicina de Ribeirão Preto USP, 2013-2015.

\begin{tabular}{lcc}
\hline \hline Características clínicas & N & \% \\
\hline Diagnóstico primário (localização do câncer) & & 40,9 \\
Mama & 9 & 18,2 \\
Próstata & 4 & 13,6 \\
Pulmão & 3 & 13,6 \\
Cólon e reto & 3 & 4,5 \\
Esôfago & 1 & 4,5 \\
Células renais claras & 1 & 4,5 \\
Bexiga & 1 & \\
Procedimento cirúrgico & & 63,6 \\
Abordagem vertebral posterior (AVP) & 14 & 22,7 \\
Cifoplastia & 5 & 9,1 \\
Abordagem vertebral anterior (AVA) & 2 & 4,5 \\
AVA+AVP & 1 & 36,4 \\
Nível lesão metastática & & 31,8 \\
Lombar & 8 & 13,6 \\
Torácica & 7 & 13,6 \\
Cervical & 3 & 4,5 \\
Toracolombar & 3 & - \\
Lombo-sacra & 1 & - \\
Sacral & - & 50 \\
Cervico-torácica & - & 22,7 \\
Tratamento pós-operatório & & 18,2 \\
Nenhum & 11 & 9,1 \\
Radioterapia & 5 & \\
Quimioterapia & 4 & \\
Quimioterapia + Radioterapia & 2 & \\
\hline
\end{tabular}




\subsection{Avaliação da Qualidade de Vida Relacionada à Saúde dos pacientes com metástase vertebral (dimensões do EORTC QLQ-C30)}

De acordo com as diretrizes do EORTC, os escores do QLQ-C30 são obtidos em uma escala de zero a cem. Na escala de Estado de Saúde Global e Qualidade de Vida, que avaliam a percepção do paciente quanto à sua saúde e qualidade de vida, quanto maior for o escore dessa escala, melhor será o estado de saúde deste indivíduo. Na Escala Funcional, zero indica o pior grau de funcionalidade e cem o melhor. O contrário ocorre na Escala de Sintomas, onde o número cem indica maior prevalência de sintomas e zero a ausência dos mesmos. Completando com a Escala de Dificuldade Financeira, um escore elevado indica pior qualidade de vida (FAYERS; BOTTOMLEY, 2002).

Analisando a Tabela 3, observa-se que com relação à escala do Estado de Saúde Global/Qualidade de Vida (ESG/QV), os pacientes na fase pré-operatória (T0) apresentavam um escore baixo, com média de 46,97 (DP = 28,12). Após a realização da cirurgia, uma nova análise da ESG/QV mostrou uma melhora acentuada aos dois meses (T1) com média de 57,35 (DP $=25,15)$. Houve manutenção desta melhora ao se analisar o questionário aos quatro meses $(\mathbf{T} 2)$ (média = 52,38; $(\mathrm{DP}=22,03)$ ) e aos seis meses $(\mathbf{T} 3)$ (média = 57,64; $(\mathrm{DP}=$ 23,96)).

Avaliando a Escala Funcional que é composta por cinco domínios (Função Física, Desempenho de Papel, Função Emocional, Função Cognitiva e Função Social), notamos que na fase pré-operatória as médias de quatro das cinco variáveis analisadas eram bem baixas, três delas abaixo de 50. Comparando-se estas médias iniciais do T0 com os tempos T1, T2 e T3, para todos os domínios houve uma melhora significativa das médias, denotando uma melhora da Escala Funcional após o procedimento cirúrgico, exceção apenas para o período de quatro meses da Função Emocional. Os maiores ganhos absolutos foram alcançados na Função Física e Desempenho de Papel, sobretudo no período T3. A categoria que teve menor influência com o procedimento cirúrgico foi a Função Emocional, em parte porque no préoperatório ela já apresentava uma média de 76,51.

Analisando os resultados para a Escala de Sintomas, composta pelas variáveis Fadiga, Náuseas e Vômitos, Dor, Dispneia, Perda de Apetite, Insônia, Diarreia e Constipação, notamos que a Dor é o sintoma mais importante, seguido por um grupo de quatro variáveis composto por Fadiga, Perda de Apetite, Insônia e Constipação. Pouco importantes na fase do pré-operatório foram as Náuseas e Vômitos, Dispneia e Diarreia, exemplificando bem a 
população estudada, composta por pacientes com algum tipo de câncer com metástase na coluna vertebral sem estar recebendo quimioterapia ou radioterapia no momento préoperatório. Levando-se em consideração os oito sintomas apresentados, o maior benefício encontrado com a realização do procedimento cirúrgico foi em relação à Dor, que apresentou melhoras em números absolutos nos três momentos avaliados (T1, T2 e T3). Houve melhora significativa aos seis meses da Fadiga, Náuseas e Vômitos, Dispneia, Perda de Apetite, Insônia, Diarreia e Constipação.

Finalizando a análise do instrumento EORTC QLQ-C30, o escore da Escala de Dificuldade Financeira manteve-se estável nos momentos T1, T2 e T3 quando comparado com o T0, confirmando as dificuldades financeiras enfrentadas ao longo desse tratamento oncológico (Tabela 3).

Tabela 3 - Média e desvio-padrão dos escores das escalas do instrumento EORTC QLQ-C30 apresentados pelos pacientes $(\mathrm{n}=22)$ com metástase vertebral, do Hospital das Clínicas da Faculdade de Medicina de Ribeirão Preto-USP, nos tempos avaliados (T0, T1, T2 e T3), 2013-2015.

\begin{tabular}{|c|c|c|c|c|c|c|c|c|}
\hline \multirow[t]{2}{*}{ Escalas e Sintomas } & \multicolumn{2}{|c|}{$\begin{array}{c}\text { T0 - Pré-operatório } \\
(\mathrm{n}=22)\end{array}$} & \multicolumn{2}{|c|}{$\begin{array}{c}\text { T1 }-2 \text { meses } \\
\quad(n=17)\end{array}$} & \multicolumn{2}{|c|}{$\begin{array}{c}\text { T2 - } 4 \text { meses } \\
(n=14)\end{array}$} & \multicolumn{2}{|c|}{$\begin{array}{c}\text { T3-6 meses } \\
(n=12)\end{array}$} \\
\hline & Média & DP & Média & DP & Média & DP & Média & DP \\
\hline ESG/QV & 46,97 & 28,12 & 57,35 & 25,15 & 52,38 & 22,03 & 57,64 & 23,96 \\
\hline Função Física & 24,85 & 26,52 & 35,69 & 34,77 & 39,05 & 33,16 & 47,22 & 36,45 \\
\hline Desempenho de Papel & 14,39 & 29,68 & 47,06 & 40,06 & 48,81 & 37,25 & 50,00 & 36,24 \\
\hline Função Cognitiva & 76,51 & 27,53 & 89,21 & 14,36 & 84,52 & 16,62 & 80,89 & 14,79 \\
\hline Função Social & 40,15 & 31,14 & 53,92 & 37,51 & 48,81 & 37,25 & 58,33 & 36,58 \\
\hline Função Emocional & 56,44 & 34,11 & 57,35 & 30,88 & 50,00 & 20,93 & 62,50 & 27,41 \\
\hline Fadiga & 48,99 & 19,60 & 32,02 & 26,02 & 40,48 & 29,43 & 36,11 & 23,75 \\
\hline Náuseas/vômitos & 15,15 & 21,77 & 19,61 & 16,91 & 50,00 & 94,28 & 9,72 & 11,14 \\
\hline Dor & 83,33 & 18,54 & 35,29 & 32,74 & 45,24 & 36,06 & 44,44 & 26,91 \\
\hline Dispneia & 16,67 & 32,12 & 5,88 & 24,25 & 11,90 & 21,11 & 11,11 & 21,71 \\
\hline Insônia & 51,51 & 44,52 & 35,29 & 41,62 & 26,19 & 35,03 & 30,55 & 33,21 \\
\hline Perda de apetite & 42,42 & 40,08 & 47,06 & 45,73 & 50,00 & 44,81 & 30,55 & 38,82 \\
\hline Constipação & 56,06 & 42,89 & 33,33 & 39,09 & 14,28 & 25,20 & 8,33 & 15,07 \\
\hline Diarreia & 6,06 & 16,70 & 17,65 & 39,29 & 9,52 & 20,37 & 2,78 & 9,62 \\
\hline Dificuldade financeira & 53,03 & 44,44 & 41,18 & 41,71 & 59,52 & 41,71 & 50,00 & 38,92 \\
\hline
\end{tabular}


As tabelas 4, 5 e 6 apresentam o Teste $t$ de Student pareado das escalas do instrumento EORTC QLQ-C30, comparando o T0 respectivamente com os momentos T1 (dois meses), T2 (quatro meses) e T3 (seis meses), considerando significantes valores de $\mathrm{p}<0,05$.

Na tabela 4, observamos uma melhora estatisticamente significativa para o Estado de Saúde Global/Qualidade de Vida (EGS/QV), Desempenho de Papel, Fadiga, Dor, Insônia e Constipação, curiosamente não sendo retratada uma melhora na Função Física dos pacientes. Dos 22 pacientes incluídos no estudo, cinco já haviam falecido antes mesmo de serem avaliados após dois meses do procedimento cirúrgico.

Tabela 4 - Média e desvio-padrão da diferença entre os pares de avaliação do momento T0 e o T1, valor p do Teste $t$ de Student pareado dos escores do instrumento EORTC QLQ-C30 apresentados pelos pacientes $(n=17)$ com metástase vertebral, do Hospital das Clínicas da Faculdade de Medicina de Ribeirão Preto-USP, 2013-2015.

\begin{tabular}{lcccc}
\hline \multicolumn{1}{c}{ Escalas e Sintomas } & \multicolumn{3}{c}{ T0 x T1 } \\
& Média & DP & Teste $\boldsymbol{~}$ & $\boldsymbol{p}$ \\
\hline EGS/QV & 16,18 & 30,97 & 2,15 & $\mathbf{0 , 0 4 7}$ \\
Função Física & 8,23 & 36,86 & 0,92 & 0,371 \\
Desempenho de Papel & 32,35 & 39,30 & 3,40 & $\mathbf{0 , 0 0 4}$ \\
Função Cognitiva & 10,78 & 22,00 & 2,02 & 0,060 \\
Função Social & 12,75 & 43,11 & 1,22 & 0,241 \\
Função Emocional & 0,49 & 29,97 & 0,06 & 0,947 \\
Fadiga & 15,03 & 24,20 & 2,56 & $\mathbf{0 , 0 2 1}$ \\
Náuseas/vômitos & 2,94 & 19,75 & 0,06 & 0,548 \\
Dor & 48,04 & 34,30 & 5,78 & $\mathbf{0 , 0 0 0 1}$ \\
Dispneia & 1,96 & 32,21 & 0,25 & 0,805 \\
Insônia & 13,73 & 37,38 & 1,51 & $\mathbf{0 , 0 2 7}$ \\
Perda de Apetite & 11,76 & 40,72 & 1,20 & 0,251 \\
Constipação & 23,53 & 45,28 & 2,14 & $\mathbf{0 , 0 4 8}$ \\
Diarreia & 13,73 & 37,38 & 1,51 & 0,150 \\
Dificuldade Financeira & 7,84 & 34,42 & 0,94 & 0,361 \\
\hline \hline
\end{tabular}

Ao se analisar o momento $\mathbf{T} 2$ do estudo (Tabela 5), notamos que apenas quatro categorias da Escala de Sintomas mantiveram resultados estatisticamente significativos (Desempenho de Papel, Dor, Insônia e Constipação), sendo que nenhum dos domínios da Escala Funcional apresentou benefícios estatísticos reais. Aos quatro meses, oito pacientes haviam falecidos. 
Tabela 5 - Média e desvio-padrão da diferença entre os pares de avaliação do momento T0 e o T2, valor p do Teste $t$ de Student pareado para os escores do instrumento EORTC QLQC30 apresentados pelos pacientes $(n=14)$ com metástase vertebral, do Hospital das Clínicas da Faculdade de Medicina de Ribeirão Preto-USP, 2013-2015.

\begin{tabular}{lcccc}
\hline \multicolumn{1}{c}{ Escalas e sintomas } & & \multicolumn{3}{c}{ T0 x T2 } \\
& Média & DP & Teste & $\boldsymbol{p}$ \\
\hline EGS/QV & 10,12 & 37,15 & 1,02 & 0,273 \\
Função Física & 13,81 & 27,73 & 1,86 & 0,085 \\
Desempenho de Papel & 32,14 & 37,25 & 3,23 & $\mathbf{0 , 0 0 7}$ \\
Função Cognitiva & 7,14 & 19,30 & 4,00 & 0,190 \\
Função Social & 10,71 & 47,86 & 0,84 & 0,417 \\
Função Emocional & 4,16 & 27,30 & 0,57 & 0,578 \\
Fadiga & 8,73 & 23,54 & 1,36 & 0,189 \\
Náuseas/vômitos & 29,76 & 81,70 & 1,36 & 0,196 \\
Dor & 38,09 & 37,23 & 3,83 & $\mathbf{0 , 0 0 2}$ \\
Dispneia & 2,38 & 33,24 & 0,27 & 0,793 \\
Insônia & 30,95 & 44,27 & 2,61 & $\mathbf{0 , 0 2 1}$ \\
Perda de Apetite & 14,29 & 31,25 & 1,71 & 0,111 \\
Constipação & 38,09 & 59,71 & 2,39 & $\mathbf{0 , 0 3 3}$ \\
Diarreia & 4,76 & 22,09 & 0,80 & 0,435 \\
Dificuldade Financeira & 0,00 & 43,36 & 0,00 & 1,000 \\
\hline \hline
\end{tabular}

Aos seis meses, última fase do estudo (Tabela 6), mantiveram resultados estatisticamente significativos os itens Desempenho de Papel, Dor, Insônia e Constipação, acrescidos da Fadiga e pela primeira vez da Função Cognitiva. O estudo se encerrou com dez óbitos no total.

Tabela 6 - Média e desvio-padrão da diferença entre os pares de avaliação do momento T0 e o T3, valor p do Teste t de Student pareado dos escores do instrumento EORTC QLQ-C30 apresentados pelos pacientes $(\mathrm{n}=12)$ com metástase vertebral, do Hospital das Clínicas da Faculdade de Medicina de Ribeirão Preto-USP, 2013-2015.

\begin{tabular}{lcccc}
\hline \multicolumn{1}{c}{ Escalas e sintomas } & Média & DP & Teste & T x T3 \\
\hline EGS/QV & 17,36 & 35,26 & 1,71 & 0,116 \\
Função Física & 17,78 & 33,68 & 1,82 & 0,095 \\
Desempenho de Papel & 30,56 & 38,82 & 2,72 & $\mathbf{0 , 0 2 0}$ \\
Função Cognitiva & 13,89 & 19,89 & 2,42 & $\mathbf{0 , 0 3 4}$ \\
Função Social & 16,67 & 51,25 & 1,13 & 0,284 \\
Função Emocional & 6,25 & 27,55 & 0,79 & 0,449 \\
Fadiga & 12,96 & 16,97 & 2,65 & $\mathbf{0 , 0 2 3}$ \\
Náuseas/vômitos & 8,33 & 23,03 & 1,25 & 0,236 \\
Dor & 38,89 & 26,91 & 5,00 & $\mathbf{0 , 0 0 0 1}$ \\
Dispneia & 0,00 & 34,82 & 0,00 & 1,000 \\
Insônia & 30,56 & 45,97 & 2,30 & $\mathbf{0 , 0 4 2}$ \\
Perda de Apetite & 2,78 & 30,01 & 0,32 & 0,754 \\
Constipação & 44,44 & 43,42 & 3,55 & $\mathbf{0 , 0 0 5}$ \\
Diarreia & 0,00 & 14,21 & 0,00 & 1,000 \\
Dificuldade Financeira & 5,56 & 44,57 & 0,43 & 0,674 \\
\hline \hline
\end{tabular}


A melhora da dor ficou evidenciada nos resultados dos escores do instrumento EORTC QLQ-C30 em todos os três momentos avaliados. Na tabela 7, temos os resultados da Escala de Dor de Faces Revisada (FPS-R). No T0, 72,8\% dos pacientes apresentavam um escore igual ou maior que seis para dor, sendo 31,9\% escore oito ou dez. Após o procedimento cirúrgico, nenhum paciente voltou a apresentar dor maior que seis, sendo que no T1 aproximadamente 30\% encontravam-se completamente assintomáticos. No momento T3, a porcentagem de pacientes assintomáticos alcançou 25\% e nove dos doze pacientes ainda vivos apresentavam escore de dor de no máximo dois. Esta análise corrobora o que foi visto no questionário do EORTC QLQ-C30 considerando-se este sintoma.

Tabela 7 - Frequência e porcentagem da Escala de Dor de Faces Revisada (FPS-R) dos pacientes $(n=22)$ com metástase vertebral, do Hospital das Clínicas da Faculdade de Medicina de Ribeirão Preto-USP, 2013-2015.

\begin{tabular}{ccccc}
\hline \hline FPS-R & T0 - n (\%) & T1 - n (\%) & T2-n (\%) & T3-n (\%) \\
\hline $\mathbf{0}$ & $0(0)$ & $5(29,4)$ & $2(14,3)$ & $3(25,0)$ \\
$\mathbf{2}$ & $3(13,6)$ & $2(11,8)$ & $7(50,0)$ & $6(50,0)$ \\
$\mathbf{4}$ & $3(13,6)$ & $5(29,4)$ & $1(7,1)$ & $2(16,7)$ \\
$\mathbf{6}$ & $9(40,9)$ & $5(29,4)$ & $4(28,6)$ & $1(8,3)$ \\
$\mathbf{8}$ & $3(13,6)$ & $0(0)$ & $0(0)$ & $0(0)$ \\
$\mathbf{1 0}$ & $4(18,3)$ & $0(0)$ & $0(0)$ & $0(0)$ \\
Total & $\mathbf{2 2 ( 1 0 0 )}$ & $\mathbf{1 7 ( 1 0 0 )}$ & $\mathbf{1 4}(\mathbf{1 0 0})$ & $\mathbf{1 2 ( 1 0 0 )}$ \\
\hline \hline
\end{tabular}

A tabela 8 mostra a relação das médias, medianas e desvios-padrão do item dor do instrumento EORTC QLQ C-30, da Escala de Dor de Faces Revisada e do Índice de Barthel em todos os momentos do estudo. Nota-se uma diminuição dos escores de dor em ambas as escalas que mensuram este sintoma, sendo que na FPS-R esta melhora é contínua e progressiva, atingindo seu ápice aos seis meses de pós-operatório. Os resultados do Índice de Barthel, instrumento utilizado para avaliação do desempenho das atividades de vida diária (AVDs), mostram uma melhora de aproximadamente 10\% no T1 e T2. Aos seis meses, há um aumento da média deste índice, alcançando quase 30\% de ganho nas AVDs dos pacientes. 
Tabela 8 - Análise descritiva da variável dor do instrumento EORTC QLQ-C30, da Escala de Dor de Faces Revisada (FPS-R) e do Índice de Barthel dos pacientes $(\mathrm{n}=22)$ com metástase vertebral, do Hospital das Clínicas da Faculdade de Medicina de Ribeirão Preto-USP, 20132015.

\begin{tabular}{lccccc}
\hline \hline \multicolumn{1}{c}{ Variável } & Valor Mínimo & Valor Máximo & Média & Mediana & DP \\
\hline Dor no T0 & 33 & 100 & 83,33 & 83,33 & 18,54 \\
Dor no T1 & 0 & 100 & 35,29 & 33,33 & 32,74 \\
Dor no T2 & 0 & 100 & 45,24 & 33,33 & 36,06 \\
Dor no T3 & 17 & 100 & 44,44 & 33,33 & 26,90 \\
FPSR no T0 & 2 & 10 & 6,18 & 6,00 & 2,54 \\
FPSR no T1 & 0 & 6 & 3,18 & 4,00 & 2,45 \\
FPSR no T2 & 0 & 6 & 3,00 & 2,00 & 2,18 \\
FPSR no T3 & 0 & 6 & 2,17 & 2,00 & 1,80 \\
Barthel no T0 & 20 & 100 & 61,59 & 67,50 & 26,61 \\
Barthel no T1 & 5 & 100 & 68,82 & 80,00 & 32,24 \\
Barthel no T2 & 0 & 100 & 67,50 & 87,50 & 37,56 \\
Barthel no T3 & 25 & 100 & 79,58 & 95,00 & 28,96 \\
\hline \hline
\end{tabular}

A tabela 9 demonstra comparações do momento pré-operatório (T0) com os três períodos subsequentes, T1, T2 e T3, levando-se em consideração o item dor do Instrumento de Qualidade de Vida EORTC QLQ-C30, a Escala de Dor de Faces Revisada e o Índice de Barthel que avalia as AVDs. Utilizando o Teste t de Student pareado, observamos que o item Dor e a FPS-R foram estatisticamente significativos nos três momentos pós-cirúrgicos, demonstrando melhora dos sintomas com o procedimento. Já a análise do Índice de Barthel não demonstrou benefício estatístico em nenhum dos períodos mencionados, não caracterizando uma melhora nas AVDs com esta ferramenta estatística utilizada. 
Tabela 9 - Valor de p do Teste $t$ de Student pareado dos escores da dor avaliada por duas escalas (EORTC QLQ-C30 e FPS-R) e da capacidade funcional (Índice de Barthel) comparando o T0 com os três tempos subsequentes T1 $(n=17), \mathbf{T} 2(n=14)$ e T3 $(n=12)$ dos pacientes com metástase vertebral, do Hospital das Clínicas da Faculdade de Medicina de Ribeirão Preto-USP, 2013-2015.

\begin{tabular}{lc}
\hline \multicolumn{1}{c}{ Variável } & $\boldsymbol{p}$ \\
\hline Dor no T0 x Dor no T1 & 0,0001 \\
Dor no T0 x Dor no T2 & 0,002 \\
Dor no T0 x Dor no T3 & 0,0001 \\
FPSR no T0 x FPSR no T1 & 0,006 \\
FPSR no T0 x FPSR no T2 & 0,008 \\
FPSR no T0 x FPSR no T3 & 0,002 \\
Barthel no T0 x Barthel no T1 & 0,488 \\
Barthel no T0 x Barthel no T2 & 0,829 \\
Barthel no T0 x Barthel no T3 & 0,303 \\
\hline \hline
\end{tabular}

Esses resultados demonstram que após a cirurgia houve um incremento da Qualidade de Vida Relacionada à Saúde desses indivíduos, sobretudo pela diminuição dos sintomas apresentados no pré-operatório, com destaque para a melhora significativa da dor. 
DISCUSSÃO 


\section{DISCUSSÃO}

A caracterização sociodemográfica dos pacientes com metástase vertebral que compuseram a amostra deste estudo foi composta igualitariamente entre homens e mulheres, sendo que pouco mais da metade dos participantes apresentavam idade igual ou inferior a 60 anos (54,5\%). Na literatura, encontramos uma alta incidência de metástase vertebral em indivíduos de 40 a 65 anos de idade que corresponde ao período de maior risco de desenvolvimento do câncer, sobretudo para o câncer de mama que é o mais prevalente no sexo feminino, excetuando-se os cânceres de pele não melanoma (PETTEYS; SCIUBBA; GOKASLAN, 2009). Se considerarmos todas as faixas etárias, veremos um aumento na prevalência de casos de metástase vertebral em homens, pois após os 65 anos há um aumento importante nos casos de câncer de próstata, e este em números absolutos apresenta uma maior prevalência quando comparado com o câncer de mama em mulheres (SCIUBBA; GOKASLAN, 2006; PERRIN; LAXTON, 2004). Segundo dados da estimativa bianual realizada pelo Instituto Nacional de Câncer do Brasil (INCA), para o biênio 2014/2015 o número de casos novos de câncer de próstata é de 69 mil/ano e os de câncer de mama 57 mil/ano (INCA, 2014).

O câncer acomete pessoas de todas as classes sociais, porém quando afeta indivíduos com baixo nível de escolaridade e condições socioeconômicas precárias, sendo esta a realidade da maioria das famílias brasileiras, o impacto da doença é ainda mais grave. Esses pacientes e seus familiares já se encontram numa condição de vulnerabilidade social, enfrentando dificuldades de acesso a bens e serviços para satisfação de suas necessidades básicas. Neste estudo, dos 22 pacientes, apenas dois apresentavam curso superior completo, quase $80 \%$ ganhavam até dois salários mínimos por mês, e todos, devido aos problemas de saúde que apresentavam, encontravam-se aposentados ou afastados de suas atividades laborais, situações encontradas em outras referências bibliográficas produzidas no nosso país (RODRIGUES; FERREIRA, 2010).

As pessoas com doença crônica e/ou incapacitante demandam cuidados específicos. Tais cuidados, sejam parciais ou completos, na maioria das vezes é assumido pelo companheiro(a) ou, se não há esta pessoa, o paciente normalmente é encaminhado para uma casa de repouso. Esta possibilidade de ter um cuidador ao lado permite uma melhor adaptação à situação de enfermidade e a torna menos sofrida, dolorosa e estressante. Neste estudo, $59,1 \%$ dos indivíduos viviam acompanhados. 
Apesar de uma expectativa maior de diagnósticos de metástase na população masculina devido uma maior incidência de casos de câncer de próstata, no presente estudo esta prevalência foi igual entre homens e mulheres. Na amostra de 22 pacientes, nove pacientes tinham o diagnóstico de câncer de mama (40,9\%), explicando o aumento proporcional de casos no sexo feminino. Na sequência da prevalência por sítios tumorais primário vieram o câncer de próstata (18,2\%), o câncer de pulmão (13,6\%) e o câncer colorretal (13,6\%). Este dado epidemiológico também é visto em outros estudos da literatura (ONIMUS et al., 1996; ABDU; PROVENCHER, 1998; SOLBERG; BREMMES, 1999). Uma provável explicação da inversão da prevalência de metástase em coluna vertebral entre o câncer de próstata e o câncer de mama pode ser devido à indolência da evolução do quadro metastático do câncer de próstata. Este tumor pode ser controlado através de manobras hormonais com o intuito de diminuir a produção de testosterona (orquiectomia ou uso de agonistas do receptor do hormônio liberador do hormônio luteinizante) ou mesmo através da indicação de radioterapia para controle das metástases ósseas previamente à cirurgia da coluna (SEIDENFELD et al., 2000).

Quanto ao segmento da coluna vertebral acometido pela lesão metastática, houve um predomínio da região torácica, seguido pela região lombo-sacra e região cervical, dados que corroboram os encontrados na literatura (MEYER; SINGH; JENKINS, 2010; RUFF; RUFF; WANG, 2007; FALICOV et al., 2006). Sabemos que existem três maneiras principais de acontecer a disseminação tumoral para os ossos da coluna vertebral: via hematogênica, disseminação direta e via fluido cerebroespinhal. Especial importância apresenta a via hematogênica, sobretudo através dos ricos plexos venosos que acompanham a irrigação de toda a coluna vertebral (ROSS et al., 2005). Segundo os autores Meyer; Singh; Jenkins (2010) e Ruff; Ruff; Wang (2007), a maior prevalência de comprometimento da coluna torácica correlaciona-se à distribuição relativa do fluxo sanguíneo para esta região da coluna e a maior presença de massa óssea deste segmento. Isto corrobora a teoria das formas de disseminação das células tumorais para a coluna vertebral.

Na perspectiva oncológica, a compressão medular metastática gera sintomas como dor, déficits neurológicos e incontinência vesical ou intestinal. Estes sintomas reduzem de maneira importante a qualidade de vida do paciente e nestas situações o tratamento clínico normalmente não é eficiente. Embora a cirurgia para descompressão medular metastática seja considerada paliativa, ela pode trazer potencial benefício ao diminuir a dor ou impedir a progressão de um déficit neurológico do paciente. Atingindo alguns destes objetivos, será possível atribuir uma melhora na qualidade de vida destes indivíduos. 
As técnicas cirúrgicas mais antigas de descompressão medular sem estabilização da coluna vertebral traziam resultados ruins. Assim, no passado, a radioterapia era eleita a modalidade de tratamento mais utilizada para controle de dor da metástase vertebral. Evidências mais recentes têm mostrado que os métodos cirúrgicos atuais, incluindo acessos anterior e posterior com estabilização da coluna vertebral, trazem de fato melhores resultados em relação à radioterapia como método isolado de tratamento (CHOI et al., 2010).

A escolha de cada abordagem depende da localização do tumor, do tipo de reconstrução requerida, das comorbidades apresentadas pelos pacientes e da extensão da doença no segmento vertebral mais acometido. O procedimento mais utilizado neste estudo foi a abordagem vertebral posterior, ocorrendo em mais de $60 \%$ dos casos. A opção desta escolha correlaciona-se com a maior prevalência do acometimento dos níveis torácicos e lombares que juntos respondiam por quase 82\% das lesões. Diversos estudos na literatura demonstram que nestes dois níveis vertebrais, o acesso posterior é o mais indicado. (RODRIGUES et al., 2011; IBRAHIM et al., 2008; WITHAM et al., 2006; STEINMETZ; MEKHALL; BENZEL, 2001).

Na fase pós-operatória, os pacientes podiam ou não ser submetidos a tratamentos adjuvantes tais como radioterapia, quimioterapia ou a combinação das duas modalidades. A opção por um destes dois tipos de tratamento depende, sobretudo, do sítio primário do tumor e dos tratamentos prévios que os pacientes porventura já haviam recebido. Quase $60 \%$ dos pacientes no estudo tinham o diagnóstico de câncer de mama ou de câncer de próstata. Tais neoplasias apresentam um arsenal terapêutico muito extenso e uma expectativa de vida relativamente longa, mesmo se tratando de uma doença metastática (BERNARD-MARTY; CARDOSO; PICCART, 2004). Justifica-se assim, o achado que 50\% dos pacientes deste estudo foram submetidos a algum tipo de tratamento adjuvante. (PATCHELL et al., 2005).

O tratamento oferecido ao paciente com metástase vertebral tem como finalidade melhorar os sintomas que o prejudicam no desempenho de suas funções diárias, interferindo diretamente na sua Qualidade de Vida. O estudo de Falicov et al. (2006) avaliou o impacto da intervenção cirúrgica na Qualidade de Vida de pacientes com metástase vertebral até um ano após o procedimento cirúrgico. O autor utilizou o mesmo instrumento de Qualidade Vida Relacionado à Saúde (EORTC QLQ-C30) desta pesquisa, e encontrou diferenças significativas apenas para a Escala de Estado de Saúde Global/Qualidade de Vida (ESG/QV) ( $p=0,013)$ e Dor $(p=0,0016)$ até o sexto mês. A capacidade funcional destes pacientes foi avaliada através da escala de desempenho físico ECOG Performance Status (Eastern Cooperative Oncology Group). A análise desta variável em relação ao procedimento cirúrgico 
realizado não mostrou ganhos significativos. Estes resultados confirmam a importância central da dor na avaliação da Qualidade de Vida Global do paciente oncológico metastático.

Assim como no estudo de Falicov et al. (2006), o artigo de Quan et al. (2011) também avaliou uma população de pacientes nas mesmas condições clínicas e encontrou resultados semelhantes. Para os itens ESG/QV e Dor, houve uma melhora significativa comparando-se o período pré-operatório com os demais momentos pós-operatórios avaliados (um, três, seis e doze meses) ( $p<0,0001)$. A capacidade funcional foi avaliada através da escala de Frankel, muito utilizada em traumatismo raquimedular. Esta escala avalia o nível do comprometimento medular, o grau de força muscular restante e o dermátomo acometido. Ela é classificada em A, B, C, D ou E, onde A corresponde à lesão completa com comprometimento tanto motor quanto sensitivo abaixo do nível da lesão e na classificação E, ausência de déficit neurológico com funções motora e sensitiva preservadas. Os resultados pós-cirurgia mostraram que quase 100\% dos pacientes apresentaram melhora ou estabilização da avaliação da escala de Frankel quando comparados aos resultados do pré-operatório.

No estudo brasileiro de Rodrigues et al. (2011), que também avaliou a Qualidade de Vida dos indivíduos antes e após o procedimento de descompressão medular causado por metástase (pré-operatório, um e seis meses), foi utilizado o instrumento SF-36 (Short-Form Health Survey). Os resultados foram estatisticamente significativos para os itens Dor ( $p=$ $0,004)$, capacidade funcional $(p<0,001)$, aspectos sociais $(p<0,001)$ e saúde mental $(p<$ 0,001), tanto para o primeiro mês do pós-operatório, quanto para o sexto mês.

Um estudo realizado na China comparou a QVRS de dois grupos de indivíduos com metástase vertebral. O grupo um (G1), composto por 46 pacientes, recebeu intervenção cirúrgica e o grupo dois (G2), composto por 50 pacientes, não recebeu qualquer intervenção, sendo considerado o grupo controle. O instrumento utilizado foi o FACT-G (Functional Assessment of Cancer Therapy-General) nos momentos pré-operatório, um, três, seis e nove meses após o procedimento cirúrgico para o G1 e para o G2, um, três, seis e nove meses após ter sido realizado o diagnóstico da lesão vertebral metastática. O G1 apresentou escores mais elevados de Qualidade de Vida ao longo do tempo em comparação ao G2, especialmente na avaliação do terceiro mês (média $=59,3$ para o G1; média $=40,1$ para o G2) $(p<0,0001)$ (WU et al., 2010).

Com base na literatura mencionada podemos afirmar que os achados do nosso estudo foram bastante semelhantes aos previamente descritos. O instrumento próprio utilizado para avaliar a Qualidade de Vida Relacionada à Saúde na população com câncer nos possibilitou visualizar com clareza os resultados apresentados por ele. Sendo a Dor o principal sintoma 
referido pelos indivíduos no T0, esta ganhou destaque pela melhora de 57,6\% no T1 e aproximadamente 46\% nos momentos T2 e T3. Mesmo no estudo de Rodrigues et al (2011) que utilizou o SF-36, que é um instrumento que avalia a Qualidade de Vida de uma maneira geral, houve também um resultado similar.

Sabemos que a capacidade funcional dos indivíduos com metástase vertebral é prejudicada pela dor e pelo déficit neurológico, afetando de maneira importante a Qualidade de Vida. No intuito de melhor compreender as limitações físicas vivenciadas no dia a dia, avaliamos as atividades de vida diária através do Índice de Barthel, que não mostrou diferença estatística em nenhum momento do estudo. Este índice não foi empregado em nenhum outro estudo dos citados acima na literatura, porém o desempenho funcional foi avaliado em todos com outras escalas. Mesmo utilizando escalas variadas, os resultados estatísticos não foram satisfatórios de uma maneira geral (QUAN et al., 2011; RODRIGUES et al., 2011; FALICOV et al., 2006).

Os resultados mostrados nos estudos que avaliaram a Qualidade de Vida Relacionada à Saúde e a capacidade funcional em indivíduos com metástase vertebral que foram submetidos à cirurgia paliativa demonstram que o alívio da dor e a possibilidade de se evitar um comprometimento neurológico definitivo foram os maiores objetivos alcançados com o tratamento proposto, possibilitando uma sobrevida, seja ela curta ou longa, com melhor Qualidade de Vida. 
CONCLUSÃO 


\section{CONCLUSÃO}

Através deste estudo, desenvolvido na cidade de Ribeirão Preto, que teve como objetivo avaliar a Qualidade de Vida Relacionada à Saúde de pacientes submetidos ao procedimento cirúrgico paliativo na coluna vertebral decorrente de metástases de sítios primários distintos, pudemos observar que a caracterização de sua amostra, apesar de um número pequeno de participantes $(\mathrm{n}=22)$, foi semelhante à de outros estudos realizados com as mesmas características e objetivos. Além disso, notamos que a frequência da incidência de tumores por sítio primário também acompanhou a tendência mundial e nacional, com destaque para os tumores de mama e próstata, que representam os tumores mais frequentes respectivamente para o sexo feminino e masculino.

No início do estudo, quando os pacientes ainda não haviam sido submetidos ao tratamento cirúrgico (T0), observamos que os domínios de QVRS mais afetados foram EGS/QV (média = 46,97), Função Física (média = 24,85), Desempenho de Papel (média = 14,39), Fadiga (média = 48,99), Dor (média = 83,33), Insônia (média = 51,51) e Constipação (média $=56,06)$.

No decorrer das avaliações do estudo, diferenças estatisticamente significativas de melhora foram encontradas no T1 para ESG/QV ( $p=0,047)$, Desempenho de Papel ( $p=$ $0,004)$, Fadiga ( $p=0,021)$, Dor $(p=0,0001)$, Insônia $(p=0,027)$ e Constipação $(p=0,048)$ quando comparados ao momento pré-cirurgia. Em T2, as diferenças foram significativas para Desempenho de Papel $(p=0,007)$, Dor $(p=0,002)$, Insônia $(p=0,021)$ e Constipação $(p=$ 0,033), e em T3, para Desempenho de Papel ( $p=0,020)$, Função Cognitiva $(p=0,034)$, Fadiga ( $p=0,023)$, Dor $(p=0,0001)$, Insônia $(p=0,042)$ e Constipação $(p=0,005)$, sempre demonstrando melhora ao se comparar com o momento T0.

A Dor, que foi avaliada através da Escala de Dor de Faces Revisada (FPS-R) e também pelo EORTC QLQ-C30, apresentou diferenças estatísticas significantes de melhora nos momentos T1, T2 e T3 quando comparados ao momento pré-operatório (T0).

Para a avaliação da capacidade funcional, não encontramos diferenças estatisticamente significativas em nenhum momento do estudo.

Estes resultados demonstram que a intervenção cirúrgica em casos de compressão medular causado por metástases proporciona uma melhora no controle da dor e como consequência traz um ganho na Qualidade de Vida Relacionada à Saúde destes pacientes. 
CONSIDERAÇÕES FINAIS 


\section{CONSIDERAÇÕES FINAIS}

As pesquisas de QVRS são imprescindíveis na área da oncologia, pois traz informações para um melhor planejamento do tratamento e reabilitação dos pacientes com câncer numa perspectiva multiprofissional. Os resultados dessa pesquisa contribuirão para o tratamento e reabilitação de pessoas com metástases na coluna vertebral. Sob a ótica do Fisioterapeuta que tem o objetivo de desenvolver, manter ou restaurar o movimento máximo e a capacidade funcional ao longo da vida, esses dados contribuem para uma atuação segura na promoção à saúde, diagnóstico precoce e tratamento dos distúrbios cinéticos funcionais provenientes das intercorrências oncológicas, com o intuito de resgatar a funcionalidade do indivíduo, por meio do diagnóstico fisioterapêutico, prescrição e execução de métodos, técnicas e recursos terapêuticos e educativos. 
REFERENNCIAS 


\section{REFERÊNCIAS}

ABDU, W.A.; PROVENCHER, M. Primary bone and metastatic tumors of the cervical spine. Spine, v. 23, n. 24, p. 2767-2777, 1998.

AARONSON, N.K. et al. The European Organization for Research and Treatment of Cancer QLQ-C30: a quality of life instrument for use in international clinical trials in oncology. $\mathbf{J}$ Natl Cancer Inst, v. 85, n. 5, p. 365-376, 1993.

ARGUELLO, F. et al. Pathogenesis of vertebral metastasis and epidural spinal cord compression. Cancer, v. 65, p. 98-106, 1990.

ANDREULA, C.; MURRONE, M. Metastatic disease of the spine. Eur Radiol, v. 15, n. 3, p. 627-32, 2005.

BARTELS, R.H.; VAN DER LINDEM, Y.M.; VAN DER GRAAF, W.T. Spinal extradural metastasis: review of current treatment options. CA Cancer J Clin, v. 58, p. 245-259, 2008.

BERNARD-MARTY, C.; CARDOSO, F.; PICCART, M.J. Facts and controversies in systemic treatment of metastatic breast cancer. Oncologist, v. 6, n. 9, p. 617-632, 2004.

BIERI, D. et al. The Faces Pain Scale for the self-assessment of the severity of pain experienced by children: development, initial validation and preliminary investigation for ratio scale properties. Pain, v. 41, p. 139-150, 1990.

BRABO, E.P. Validação para o Brasil do questionário de qualidade de vida para pacientes com câncer de pulmão (QLQ-LC13) da Organização Européia para Pesquisa e Tratamento do Câncer. 2006. Dissertação (Mestrado em Clinica Medica). Rio de Janeiro: Universidade Federal do Rio de Janeiro, 2006.

CANPONERO, R. Biologia do câncer in: CARVALHO, V. A. et al. Temas em psicooncologia. São Paulo: Summus, 2008, p. 32-39.

CELLA, D.F. Quality of life outcomes: measurement and validation. Oncology, v. 10 (Suppl), p. 233-245, 1996.

CHAICHANA, K. L. et al. Outcome following decompressive surgery for different histological types of metastatic tumors causing epidural spinal cord compression. Clinical article. J Neurosurg Spine, v. 11, n. 1, p. 56-63, 2009. 
CHOI, D. et al. Global Spine Tumor Study Group. Review of metastatic spine tumor classification and indications for surgery: the consensus statement of the Global Spine Tumor Study Group. Eur Spine J, v. 19, n. 2, p. 215-222, 2010.

COLE, J.S.; PATCHELL, R.A. Metastatic epidural spinal cord compression. Lancet Neurol, v. 7, n. 5, p. 459-466, 2008.

COLEMAN, M.P. et al. Cancer survival in Australia, Canada, Denmark, Norway, Sweden and the UK, 1995-2007 (the International Cancer Benchmarking Partnership): an analysis of population-based cancer registry data. Lancet, v. 377, p. 127-138, 2011.

COSTELLOE, C.M., et al. Imaging bone metastases in breast cancer: techniques and recommendations for diagnosis. Lancet Oncol, v. 10, n. 6, p. 606-614, 2009.

DANIEL, J.W.; VEIGA, J.C.E. Diretrizes no tratamento das metástases epidurais da coluna vertebral. Atualização. Arq Bras Neurocir, v. 26, n. 3, p. 93-110, 2007.

DALDRUP-LINK, H.E., et al. Whole-body MR imaging for detection of bone metastases in children and young adults: comparison with skeletal cintilography and FDG PET. Am J Roentgenol, v. 177, n. 1, p. 229-236, 2001.

FALAVIGNA, A. et al. Metástases do segmento torácico e lombar da coluna vertebral. Arq neuropsiquiatria, v. 65, n. 3-B, p. 889-895, 2007.

FALICOV, A. et al. Impact of surgical intervention on quality of life in patients with spinal metastases. Spine, v. 31, p. 2849-2856, 2006.

FAYERS, P. M. et al. EORTC QLQ-C30 Scoring Manual (3rd edition). Brussels: EORTC, 2001. 78p. Disponível em: http://www.eortc.be/qol/files/SCManualQLQ-C30.pdf. Acesso em: Julho/2013.

FAYERS, P.; BOTTOMLEY, A. Quality of life research within the EORTC - the EORTC QLQ-C30. European organization for research and treatment of cancer. Eur J Cancer, v. 38, n. suppl 4, p. S125-133, 2002.

FAYERS, P. M.; MACHIN, D. Quality of life. Assessment, analyses and interpretation. 2nd ed. Chichester, England: John Wiley \& Sons, 2007.

FERREL, B.R.; DOW, K.H.; GRANT, M. Measurement of the quality of life in cancer survivors. Quality of life research, v. 4, n. 6, p. 523-531, 1995. 
FERREL, B.R.; COYLE, N. Textbook of palliative nursing. 1st ed. New York: Oxford University Press, 2001.

FUJIBAYASHI, S. et al. The value of palliative surgery for metastatic spinal disease: satisfaction of patients and their families. Spine J, v. 10, n. 1, p. 42-49, 2010.

GUILLEVIN, R., et al. Spine metastasis imaging: review of the literature. J Neuroradiol, v. 34, n. 5, p. 311-321, 2007.

HAREL, R; ANGELOV, L. Spine metastases: Current treatments and future directions. European journal of cancer, v. 46, p. 2696-2707, 2010.

HEARY, R.F.; BONO, C.M. Metastatic spinal tumors. Neurosurg Focus, v. 11, n. 6, article $1,2001$.

HESSLER, C. et al. Dynamics of neurological deficit after surgical decompression of symptomatic vertebral metastases. Spine, v. 34, p. 566-571, 2009.

HICKS, C.L. et al. The Faces Pain Scale-Revised: toward a common metric in pediatric pain measurement. Pain, v. 93, p. 173-183, 2001.

HOSONO, N. et al. Orthopedic management of spinal metastases. Clin Orthop Relat Res, v. 312, p. 148-159, 1995.

IBRAHIM, A. et al. Does spinal surgery improve the quality of life for those with extradural (spinal) osseous metastases? An international multicentre prospective observational study of 223 patients. J Neurosurgery Spine, v. 8, p. 271-278, 2008.

INSTITUTO NACIONAL DO CANCER (Brasil). Estimativa 2014: incidência de câncer no Brasil. Coordenação de Prevenção e Vigilância. Rio de Janeiro, 2014. Disponível em: http://www.inca.gov.br/estimativa/2014. Citado em 08/03/2014.

INTERNATIONAL ASSOCIATION FOR HOSPICE AND PALLIATIVE CARE (IAHPC). Manual of palliative care. IAHPC, 2003. Citado em 19/11/14. Disponível em: http://www. hospicecare.com

INTERNATIONAL AGENCY FOR RESEARCH CANCER (IARC). Globocan 2012: estimated cancer incidence, mortality and prevalence worldwide in 2012. Lyon, France, 2014. Disponível em http://globocan.iarc.fr/. Citado em 08/03/2014. 
JANSSON, K.; BAUER, H.C. Survival, complications and outcome in 282 patients operated for neurological deficit due to thoracic or lumbar metastases. Eur Spine J, v. 15, p. 196-202, 2006.

JACOBS, W.B.; PERRIN, R.G. Evaluation and treatment of spinal metastases: an overview. Neurosurg Focus, v. 11, n. 6, p. e10, 2001.

JEMAL, A. et al. Cancer statistics. CA Cancer J Clin, v. 59, p. 225-249, 2009.

JOAQUIM, A.F. et al. Metástases na coluna vertebral. Rev Neurocienc, v. 15, n. 3, p. 240245, 2007.

JU, D.G. et al. Factors associated with improved outcomes following decompressive surgery for prostate cancer metastatic to the spine. Neurosurgery, v. 73, n. 4, p. 657-666, 2013.

JUNG, H.S. et al. Discrimination of metastatic from acute osteoporotic compression spinal fractures with MR imaging. Radiographics, v. 23, n. 1, p. 179-187, 2003.

KHANNA, A. J. et al. Use of magnetic resonance imaging in differentiating compartmental location of spinal tumors. American Journal of Orthopaedics, v. 35, n.10, p. 472-476, 2005.

LAUFFER, R.; MACANEIRO, C.; MYAMOTO, R. Estabilização cirúrgica posterior nas metástases da coluna toraco-lombar. Coluna, v. 3, n. 1, p. 39-43, 2004.

MACCAURO, G. et al. Physiopathology of spine metastasis. Int J Surg Oncol, v. 2011, p.18, 2011.

MAIN, C.J.; WATSON, P.J. Psychological aspects of pain. Man Ther, v. 4, n. 4, p. 203-215, 1999.

MAHONY, F.I.; BARTHEL, D.W. Functional evaluation: the Barthel Index. Md State Med J, v. 14, p. 61-65, 1965.

McCOUGHLAN, M. A necessidade de cuidados paliativos. Mundo saúde, v. 27, n.1, p. 614, 2003.

MEYER, S.A.; SINGH, H.; JENKINS, A.L. Surgical treatment of metastatic spinal tumors. Mt Sinai J Med, v. 77, n. 1, p. 124-129, 2010.

MINOSSO, J.S.M. et al. Validação no Brasil do índice de Barthel em idosos atendidos em ambulatórios. Acta Paul Enferm, v. 23, n. 2, p. 218-223, 2010. 
ONIMUS, M. et al. Results of surgical treatment of spinal thoracic and lumbar metastases. Eur Spine J, v. 5, n. 6, p. 407-411, 1996.

PATCHELL, R. A. et al. Direct decompressive surgical resection in the treatment of spinal cord compression caused by metastatic cancer: a randomised trial. Lancet, v. 366, p. 643-648, 2005.

PERRIN, R.G.; LAXTON A.W. Metastatic spine disease: epidemiology, pathophysiology and evaluation of patients. Neurosurgery Clin North America, v. 15, n. 4, p. 365-73, 2004.

PERRIN, R.G.; LIVINGSTON, K.E.; AARABI, R. Intradural extramedullary spinal metastasis: a report of 10 cases. J neurosurg, v. 56, p. 835-837, 1982.

PETTEYS, R.J.; SCIUBBA, D.M.; GOKASLAN, Z.L. Surgical management of metastatic spine disease. Seminars in Spine Surgery, v. 21, p. 86-92, 2009.

PIMENTA, C.A.M. Dor oncológica: bases para avaliação e tratamento. Mundo Saúde, v. 27, n. 1, p. 98-110, 2003.

POINTILLART, V. et al. Survival prognostic factors and clinical outcomes in patients with spinal metastases. J Cancer Res Clin Oncol, v. 137, n. 5, p. 849-856, 2011.

POLIT, D. F.; BECK, C.T. Fundamentos da pesquisa em enfermagem. Avaliação de evidências para a prática da enfermagem. 7ed. Porto Alegre: Artmed, 2011.

PUTZ, C et al. Tokuhashi prognosis score: an important tool in prediction of the neurological outcome in metastatic spinal cord compression: a retrospective clinical study. Spine, v. 33, n. 24, p. 2669-2674, 2008.

QUAN, G. M. Y. et al. Surgery improves pain, function and quality of life in patients with spinal metastases: a prospective study on 118 patients. Eur Spine J, v. 20, p. 1970-1978, 2011.

QUINN, J.A.; DEANGELIS, L. M. Neurologic emergencies in the cancer patient. Semin Oncol, v. 27, n. 3, p. 311-321, 2000.

QURAISHI, N.A.; GOKASLAN, Z.L.; BORIANI, S. The surgical management of metastatic epidural compression of the spinal cord. J Bone Joint Surg Br, v. 92, n. 8, p. 1054-1060, 2010. 
RODRIGUES, J. S. M.; FERREIRA, N. M. L. A. Caracterização do perfil epidemiológico do câncer em uma cidade do interior paulista: conhecer para intervir. Rev. Bras. de Cancerologia, v. 56, n. 4, p. 431-441, 2010.

RODRIGUES, L. M. R. et al. Qualidade de vida de pacientes submetidos a descompressão por lesão vertebral metastática. Acta ortop. bras, v. 19, n 3, p. 149-153, 2011.

ROSS, J. et al. Diagnostic imaging: Spine, vol IV. In: ROSS, J. Altona, Manitoba, Canada: Amirys, 2005, p. 1-126.

RUFF, R.L.; RUFF, S.S.; WANG, X. Persistent benefits of rehabilitation on pain and lifequality for nonambulatory patients with spinal epidural metastasis. J Rehabil Res Dev, v. 44, n. 2, p. 271-278, 2007.

SCHIFF, D. Spinal cord compression. Neurological clinics, v. 21, n. 1, p. 67- 86, 2003.

SCHICK, U; MARQUARDT, G; LORENZ, R. Intradural and extradural spinal metastases. Neurosurgery Rev, v. 24, n. 1, p. 1-5 [discussion 6-7], 2001.

SCIUBBA, D.M.; GOKASLAN, Z.L. Diagnosis and management of metastatic spine disease. Surgical Oncology, v. 15, p. 141-151, 2006.

SCIUBBA, D.M., et al. Diagnosis and management of metastatic spine disease. J Neurosurg Spine, v. 13, n. 1, p. 94-108, 2010.

SEIDENFELD, J. et al. Single-therapy androgen suppression in men with advanced prostate cancer: a systematic review and meta-analysis. Ann Intern Med, v. 7, n. 132, p. 566-577, 2000.

SILVA, F. C. J; THULER, L. C. S. Tradução e adaptação transcultural de duas escalas para avaliação da dor em crianças e adolescentes. J Pediatria, v. 84, n. 4, p. 344-349, 2008.

SODERBERG, G.L. Kinesiology: application to pathological motion. 2nd ed. Baltimore: Williams \& Wilkins, 1997.

SOLBERG, A.; BREMNES, R.M. Metastatic spinal cord compression: diagnostic delay, treatment, and outcome. Anticancer Res, v. 19, n. 1B, p. 677-684, 1999.

STEINMETZ, M.P.; MEKHAIL, A.; BENZEL, E. C. Management of metastatic tumors of the spine: strategies and operative indications. Neurosurg Focus, v. 11, n. 6, p. e2, 2001. 
TEIXEIRA, L. A.; FONSECA, C. O. De doença desconhecida a problema de saúde pública: o INCA e o controle do câncer no Brasil. Rio de Janeiro: Ministério da saúde, 2007.

TREANOR, C.; DONNELLY, M. An international review of the patterns and determinants of health service utilization by adult cancer survivors. BMC Health Services Research, v. 12, n. 316, p. 1-20, 2012.

VACCARO, A.R.; BETZ, R.R.; ZEIDMAN, S.M. Principles and Practice of Spine Surgery. New York: Elsevier, 2003, p. 213-222.

VAN DER LINDEN, Y.M. et al. Prediction of survival in patients with metastases in the spinal column: results based on randomized trial of radiotherapy. Cancer, v. 103, n. 2, p. 320328, 2005.

VRIONIS, F.D.; SMALL, J. Surgical management of metastatic spinal neoplasm. Neurosurg Focus, v. 15, p. 1-8, 2003.

WAI, E., et al. Quality of life in surgical treatment of metastatic spine disease. Spine, v. 28, p. 508-512, 2003.

WANG, J.C., et al. Single-stage postero lateral transpedicular approach for resection of epidural metastatic spine tumors involving the vertebral body with circumferential reconstruction: results in 140 patients: invited submission from the Joint Section Meeting on Disorders of the Spine and Peripheral Nerves, March 2004. J Neurosurg Spine, v.1, p. 287298, 2004.

WEDIN, R.; BAUER, H.C.; RUTQVIST, L. E. Surgical treatment for skeletal breast cancer metastases: a population-based study of 641 patients. Cancer, v. 92, n. 2, p. 257-262, 2001.

WEIGEL, B., et al. Surgical management of symptomatic spinal metastases: postoperative outcome and quality of life. Spine, v. 24, p. 2240-2246, 1999.

WHO. The WHOQOL Group. Measuring quality of life: The development of The World Health Organization Quality of Life Instrument (WHOQOL). Geneve (SZ), 1993.

WHO 2011. Global status report on non-communicable diseases 2010. Geneva: World health Organization, 2011. Disponível em: www.who.int/cancer citado em 04/12/2014.

WITHAM, T.F. et al. Surgery insight: current management of epidural spinal cord compression from metastatic spine disease. Nat ClinPractNeurol, v. 2, n. 2, p. 87-94, 2006. 
WORLD CONFEDERATION FOR PHYSICAL THERAPY (WCPT). Policy statement: Description of physical therapy.Vancouver (Canadá), 2007. Citado em 15/10/14. Disponivel em: http: www.wcpt.org/policy/

WU, J. et al. Health-related quality of life in patients with spinal metastases treated with or without spinal surgery. A prospective, longitudinal study. Cancer, v. august 15, p. 38753882, 2010.

YOUNG, R.F.; POST, E.M.; KING, G.A. Treatment of spinal epidural metastases: Randomized prospective comparison of laminectomy and radiotherapy. J Neurosurg, v. 53, p. 741-748, 1980.

ZAIRI, F. et al. Minimally invasive decompression and stabilization for the management of thoracolumbar spine metastasis. J Neurosurg Spine, v. 17, p. 19-23, 2012. 
APÊNDICES 


\section{APÊNDICES}

\section{Apêndice 1 - Termo de consentimento livre e esclarecido (TCLE)}

\section{TERMO DE CONSENTIMENTO LIVRE E ESCLARECIDO}

O Sr.(a) está sendo convidado para participar como voluntário da pesquisa. Após ser esclarecido(a) sobre as informações a seguir, no caso de aceitar fazer parte do estudo, assine ao final deste documento que está em duas vias. Uma delas é sua e a outra é do pesquisador responsável.

Título do projeto "Pacientes com metástases vertebrais submetidos ao tratamento cirúrgico: avaliação da qualidade de vida relacionada à saúde”.

Este projeto é de responsabilidade da aluna de pós-graduação pela Escola de Enfermagem de Ribeirão Preto em enfermagem fundamental Cristiane Thomaz de Aquino Exel Andrade e da Profa. Dra. NamieOkinoSawada(Av. Bandeirantes, no. 3900 - Campus Universitário telefone para contato 3602-3438 e e-mail: crisexel@usp.br).

O Comitê de Ética em Pesquisa é formado por um grupo de pessoas que avaliou este projeto para ver se apresenta algum prejuízo para quem participa da pesquisa. Se tiver dúvidas ou se sentir de alguma forma prejudicado(a) sobre os aspectos éticos dessa pesquisa, você pode entrar em contato com o Comitê de Ética em Pesquisa da Escola de Enfermagem de Ribeirão Preto USP, pelo telefone (16) 3602-3386. O objetivo do estudo é avaliar a qualidade de vida e capacidade física (atividades do dia a dia) dos pacientes com metástase óssea na coluna vertebral antes e após o tratamento cirúrgico.

Caso concorde em participar do nosso estudo, o senhor(a) será orientado(a) sobre o projeto e deverá responder algumas perguntas relacionadas à sua condição de saúde e atividades que desenvolve diariamente.

A sua participação é voluntária e consiste em permitir a consulta ao seu prontuário para obtermos outras informações sobre sua doença e tratamento. Estas informações terão caráter sigiloso, não apresentando qualquer risco ou dano à sua saúde.

OSr(a) será convidado a participar do estudo na internação pré-operatória, onde neste momento se aceitar, assinará este documento - “Termo de Consentimento Livre e Esclarecido (TCLE)” de acordo com Resolução do Conselho Nacional de Saúde (CNS) 196/96 que será entregue pela pesquisadora e em seguida responderá o primeiro questionário do estudo que é 
o denominado "antes da cirurgia”. As demais entrevistas ocorrerão em três momentos, a saber: segundo, quarto e sexto mês após o procedimento cirúrgico e ocorrerão no ambulatório do Departamento de Biomecânica, Medicina e Reabilitação do Aparelho Locomotor do HCFMRP.

O tempo da entrevista será de aproximadamente trinta minutos. Se alguma das perguntas feitas lhe causarem algum tipo de desconforto emocional (único risco relacionado à nossa pesquisa), você poderá decidir em não continuar a responder a pesquisa se assim desejar.

Caso aceite participar desta pesquisa, contamos com sua sinceridade e clareza ao falar como está a sua saúde e capacidade física no momento da entrevista.

Os resultados deste estudo nos ajudarão a conhecer as limitações físicas e qualidade de vida no pré-operatório e pós-operatório e também os resultados do tratamento cirúrgico realizados neste hospital. Futuramente poderemos propor um atendimento que ajude a melhorar a qualidade de vida e os resultados cirúrgicos.

Para confirmar o seu aceite em participar do estudo, o Sr(a) deverá assinar o Termo de Consentimento Livre e Esclarecido junto com as pesquisadoras responsáveis pelo estudo. Garantimos que a participação do $\operatorname{Sr}(a)$ não irá interferir no seu tratamento, seu direito e liberdade para recusar-se a permissão de busca no seu prontuário; e que não haverá nenhum custo para a sua participação. Asseguramos que você não será identificado e os dados obtidos da pesquisa serão publicados e divulgados em encontros com outros profissionais da saúde. Garantimos responder a qualquer dúvida ou esclarecimentos a respeito dessa pesquisa.

O Sr(a) tem a liberdade de se recusar a participar ou retirar seu consentimento em qualquer fase da pesquisa sem penalização ou prejuízo ao seu cuidado ou atendimento nessa instituição.

Nome do paciente:

Assinatura: Data:

Nome do pesquisador:

Assinatura: Data: 


\section{Apêndice 2 - Ficha de caracterização sociodemográfica}

Nome:

Registro:

Sexo:( ) Fem ( ) Masc

Cor:

Data de nascimento:

Endereço:

Telefone:

Cidade/Estado:

Estado civil:( ) Casado ( ) Solteiro ( ) Outro Especificar

Nível escolaridade:( ) Fundamental ( ) Médio ( ) Superior

Situação Profissional:

Renda mensal:

Diagnóstico Primário:

Tipo de procedimento cirúrgico:

Data:

Nível (eis) da Lesão Metastática:

Tratamento pós-operatório:

Data das coletas: 1 )

2)

3)

4) 
ANEXOS 


\section{ANEXOS}

Anexo 1 - Escala de dor de faces revisada (FPS-R)
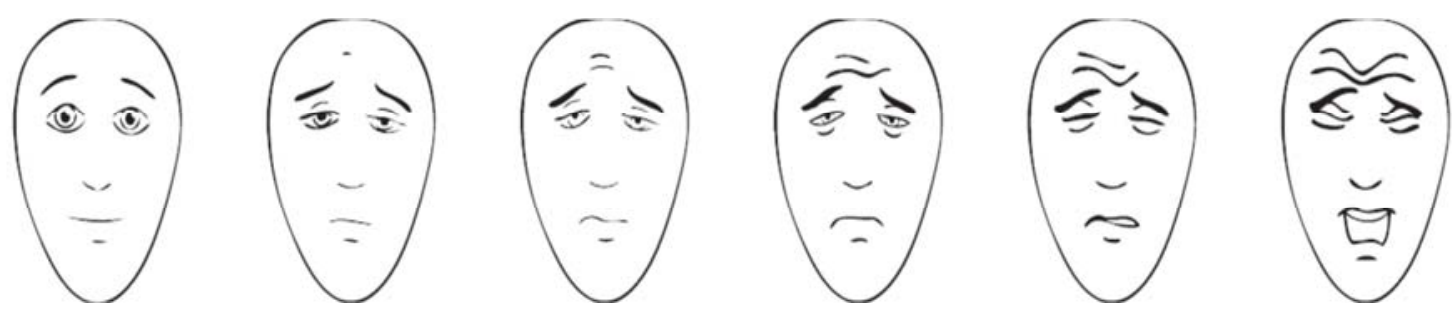


\section{Anexo 2 - Instrumento EORTCQLQ-C30}

\section{EORTC QLQ-C30 (versão 3.0.)}

Nós estamos interessados em alguns dados sobre você e sua saúde. Responda, por favor, a todas as pergunt: fazendo um círculo no número que melhor se aplica a você. Não há respostas certas ou erradas. A informaçą que você fornecer permanecerá estritamente confidencial.

Por favor, preencha suas iniciais:

Sua data de nascimento (dia, mês, ano):

Data de hoje (dia, mês, ano):

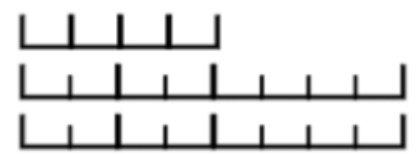

1. Você tem qualquer dificuldade quando faz grandes esforços, por exemplo carregar uma bolsa de compras pesada ou uma mala?

Não

Pouco damente

2. Você tem qualquer dificuldade quando faz uma longa caminhada?

3. Você tem qualquer dificuldade quando faz uma curta caminhada fora de casa?

4. Você tem que ficar numa cama ou na cadeira durante o dia?

5. Você precisa de ajuda para se alimentar, se vestir, se lavar ou usar o banheiro?

\section{Durante a última semana:}

6. Tem sido dificil fazer suas atividades diárias?

7. Tem sido dificil ter atividades de divertimento ou lazer?

8. Você teve falta de ar?

9. Você tem tido dor?

10. Você precisou repousar?

11. Você tem tido problemas para dormir?

12. Você tem se sentido fraco/a?

13. Você tem tido falta de apetite?

14. Você tem se sentido enjoado/a?

15. Você tem vomitado?

\section{Não Pouco}

Modera- Muito damente

3

4

4

4

4

4

4

4

4

4

4 


\section{Durante a última semana:}

16. Você tem tido prisão de ventre?

17. Você tem tido diarréia?

18. Você esteve cansado/a?

19. A dor interferiu em suas atividades diárias?

20. Você tem tido dificuldade para se concentrar em coisas, como ler jornal ou ver televisão?

21. Você se sentiu nervoso/a?

22. Você esteve preocupado/a?

23. Você se sentiu irritado/a facilmente?

24. Você se sentiu deprimido/a?

25. Você tem tido dificuldade de se lembrar das coisas?

26. A sua condição fisica ou o tratamento médico tem interferido em sua vida familiar?

27. A sua condição fisica ou o tratamento médico tem interferido em suas atividades sociais?

28. A sua condição fisica ou o tratamento médico tem the trazido dificuldades financeiras?

\section{Não Pouco Modera- Muito damente}

4

4

4

4

4

4

4

4

4

4

4

$\begin{array}{llll}1 & 2 & 3 & 4\end{array}$

$\begin{array}{llll}1 & 2 & 3 & 4\end{array}$

\section{Para as seguintes perguntas, por favor, faça um círculo em volta do número entre 1 e 7 que melhor se aplica a você.}

29. Como você classificaria a sua saúde em geral, durante a última semana?

$\begin{array}{llllllll}1 & 2 & 3 & 4 & 5 & 6 & 7\end{array}$

Péssima

Ótima

30. Como você classificaria a sua qualidade de vida geral, durante a última semana?
1
2
3
4
5
6
7

Péssima

Ótima 


\section{Anexo 3 - Autorização para uso do Instrumento EORTCQLQ-C30}

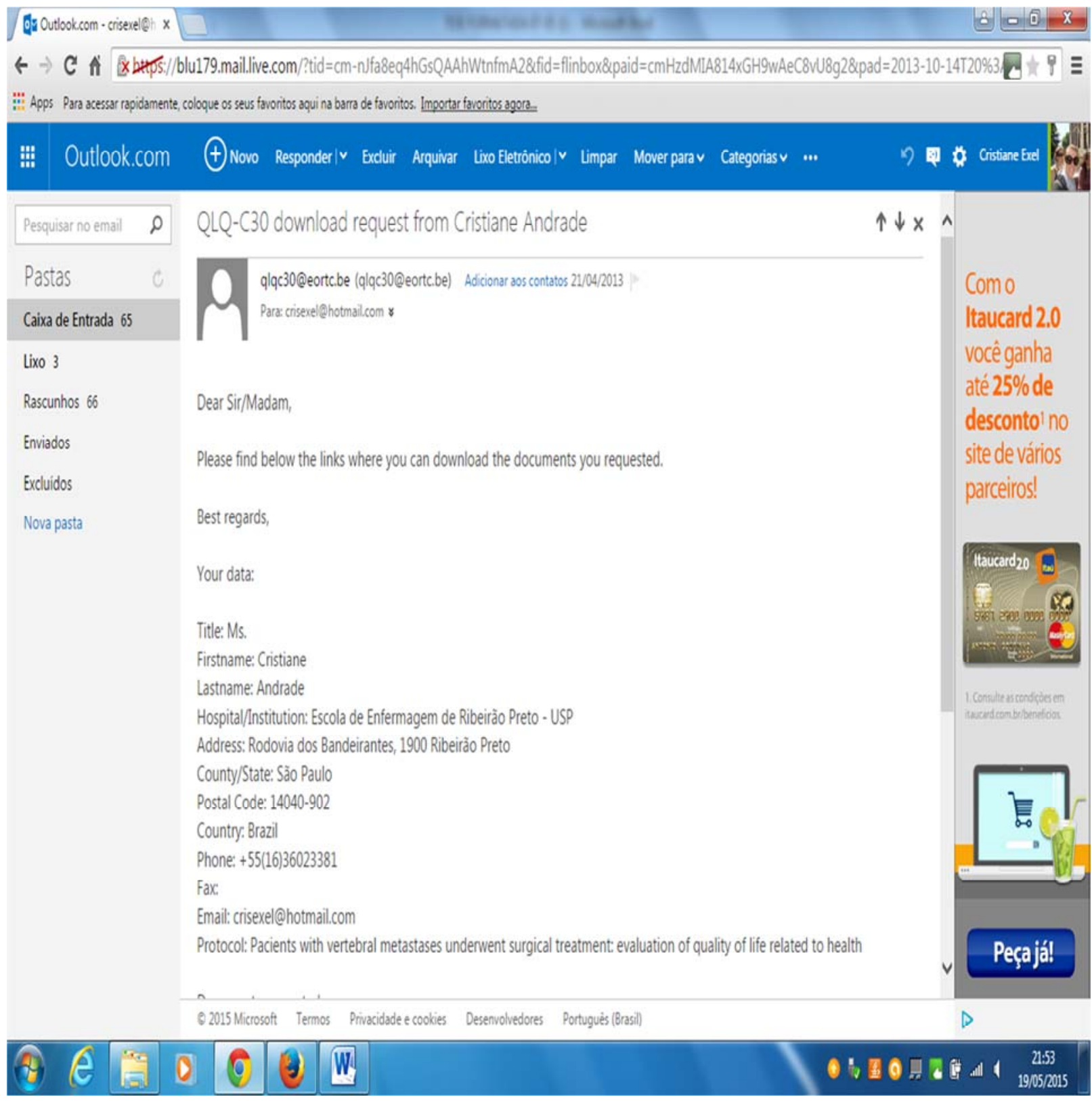




\section{Anexo 4 - Escala Funcional de Barthel}

\section{ALIMENTAÇÃO}

0 - Incapacidade

5 - precisa de ajuda para cortar, passar manteiga, et, ou dieta modificada

$\square 10$ - Independente

\section{BANHO}

0 - Dependente

5 - Independente (ou no chuveiro)

\section{ATIVIDADES ROTINEIRAS}

$\square 0$ - Precisa de ajuda com a higiene pessoal

$\square$ - Independente rosto/cabelo/dentes/barbear

\section{VESTIR-SE}

0 - Dependente

5 - Precisa de ajuda mas consegue fazer uma parte sozinho

$\square 10$ - Independente (incluindo botões, zipers, laços, etc.)

\section{INTESTINO}

$\square 0$ - Incontinente (necessidade de enemas)

$\square$ - Acidente ocasional

$\square 10$ - Continente

\section{SISTEMA URINÁRIO}

$\square 0$ - Incontinente, ou cateterizado e incapaz de manejo

5 - Acidente ocasional

$\square 10$ - Continente

\section{USO DO TOILET}

$\square$ - Dependente

5 - Precisa de alguma ajuda parcial

$\square 10$ - Independente (pentear-se, limpar-se)

\section{TRANSFERÊNCIA (DA CAMA PARA A CADEIRA E VICE VERSA)}

0 - Incapacitado, sem equilíbrio para ficar sentado

- 5 - Muita ajuda (uma ou duas pessoas, física), pode sentar

- 10 - Pouca ajuda (verbal ou física)

$\square 15$ - Independente

\section{MOBILIDADE (EM SUPERFÍCIES PLANAS)}

$\square$ - Imóvel ou $<50$ metros

5 - Cadeira de rodas independnte, incluindo esquinas, >50 metros

$\square 10$ - Caminha com ajuda de uma pessoa (verbal ou física) > 50 metros

$\square 15$ - Independente (mas pode precisar de alguma ajuda

\section{ESCADAS}

0 - Incapacitado

5 - Precisa de ajuda (verbal, física, ou ser carregado)

$\square 10$ - Independente 
Anexo 5 - Carta de Aprovação pelo Comitê de Ética em Pesquisa da EERP/USP
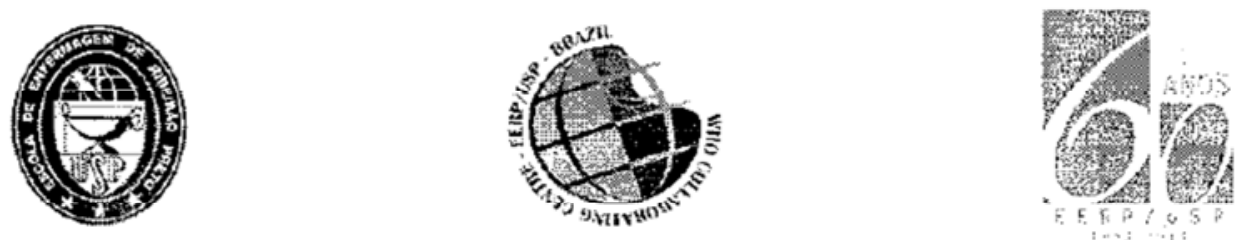

ESCOLA DE ENFERMAGEM DE RIBEIRĂO PRETO DA UNIVERSIDADE DE SĀO PAULO

Centro Colaborador da Organização Mundial da Saúde para o Desenvolvimento da Pesquisa em Enfermagem

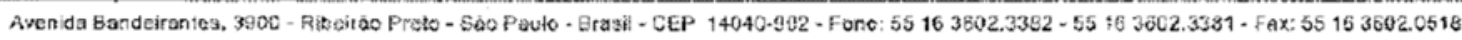
wwrwearp.uaphs - oorp@edtusp.br

\section{COMITÊ DE ÉTICA EM PESQUISA DA EERP/USP}

Of.CEP-EERP/USP - 243/2013

Ribeirăo Preto, 16 de outubro de 2013

Prezada Senhora,

Comunicamos que o projeto de pesquisa, abaixo especificado, foi analisado e considerado APROVADO AD REFERENDUM pelo Comitê de Ética em Pesquisa da Escola de Enfermagem de Ribeirão Preto da Universidade de São Paulo, em 16 de outubro de 2013.

Protocolo CAAE: 10801713.2 .0000 .5393

Projeto: Pacientes com metástases vertebrais submetidos ao tratamento cirúrgico: avaliação da qualidade de vida relacionada à saúde.

Pesquiisadores: Namie Okino Sawada

Cristiane Thomaz de Aquino Exel Andrade

Em atendimento à Resoluçăo 466/12, deverá ser encaminhado ao CEP o relatório final da pesquisa e a publicação de seus resultados, para acompanhamento, bem como comunicada qualquer intercorréncia ou a sua interrupção.

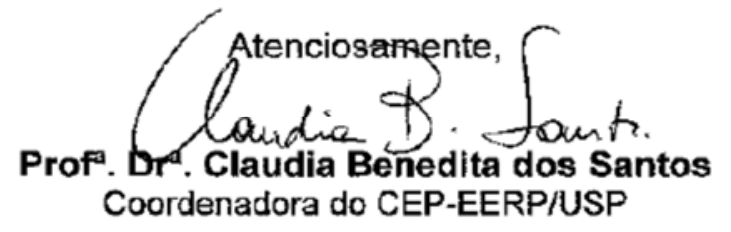

Ilma. Sra.

Profa. Dra. Namie Okino Sawada

Departamento de Enfermagem Geral e Especializada

Escola de Enfermagem de Ribeirão Preto - USP 